\title{
Venlafaxine Stimulates an MMP-9-Dependent Increase in Excitatory/Inhibitory Balance in a Stress Model of Depression
}

\author{
${ }^{\circledR}$ Seham Alaiyed, ${ }^{1,2}$ Mondona McCann, ${ }^{2,3}$ Gouri Mahajan, ${ }^{4}$ Grazyna Rajkowska, ${ }^{4}{ }^{\circledR}$ Craig A. Stockmeier, ${ }^{4}$ \\ ${ }^{-}$Kenneth J. Kellar, ${ }^{1}$ Jian Young $\mathrm{Wu}^{2,3}$ and ${ }^{\circledR}$ Katherine Conant ${ }^{2,3}$ \\ ${ }^{1}$ Department of Pharmacology, ${ }^{2}$ Department of Neuroscience, ${ }^{3}$ Interdisciplinary Program in Neuroscience, Georgetown University Medical Center, \\ Washington, DC 20007, and ${ }^{4}$ Department of Psychiatry and Human Behavior, University of Mississippi Medical Center, Jackson, Mississippi \\ 39216
}

Emerging evidence suggests that there is a reduction in overall cortical excitatory to inhibitory balance in major depressive disorder (MDD), which afflicts $\sim 14 \%-20 \%$ of individuals. Reduced pyramidal cell arborization occurs with stress and MDD, and may diminish excitatory neurotransmission. Enhanced deposition of perineuronal net (PNN) components also occurs with stress. Since parvalbuminexpressing interneurons are the predominant cell population that is enveloped by PNNs, which enhance their ability to release GABA, excess PNN deposition likely increases pyramidal cell inhibition. In the present study, we investigate the potential for matrix metalloprotease-9 (MMP-9), an endopeptidase secreted in response to neuronal activity, to contribute to the antidepressant efficacy of the serotonin/norepinephrine reuptake inhibitor venlafaxine in male mice. Chronic venlafaxine increases MMP-9 levels in murine cortex, and increases both pyramidal cell arborization and PSD-95 expression in the cortex of WT but not MMP-9-null mice. We have previously shown that venlafaxine reduces PNN deposition and increases the power of ex vivo $\gamma$ oscillations in conventionally housed mice. $\gamma$ power is increased with pyramidal cell disinhibition and with remission from MDD. Herein we observe that PNN expression is increased in a corticosterone-induced stress model of disease and reduced by venlafaxine. Compared with mice that receive concurrent venlafaxine, corticosterone-treated mice also display reduced ex vivo $\gamma$ power and impaired working memory. Autopsy-derived PFC samples show elevated MMP-9 levels in antidepressant-treated MDD patients compared with controls. These preclinical and postmortem findings highlight a link between extracellular matrix regulation and MDD.

Key words: depression; MMP; MMP-9; parvalbumin; perineuronal net; venlafaxine

\section{Significance Statement}

Reduced excitatory neurotransmission occurs with major depressive disorder, and may be normalized by antidepressant treatment. Underlying molecular mechanisms are, however, not well understood. Herein we investigate a potential role for an extracellular protease, released from neurons and known to play a role in learning and memory, in antidepressant-associated increases in excitatory transmission. Our data suggest that this protease, matrix metalloprotease-9, increases branching of excitatory neurons and concomitantly attenuates the perineuronal net to potentially reduce inhibitory input to these neurons. Matrix metalloprotease-9 may thus enhance overall excitatory/inhibitory balance and neuronal population dynamics, which are important to mood and memory.

Received 0ct. 4, 2019; revised Mar. 19, 2020; accepted Mar. 20, 2020.

Author contributions: K.C., S.A., and K.J.K. designed research; K.C., S.A., M.M., and J.Y.W. performed research; K.C., S.A., and M.M. analyzed data; K.C., S.A., C.A.S., K.J.K., and J.Y.W. edited the paper; K.C. and S.A. wrote the paper; G.M., G.R., and C.A.S. contributed unpublished reagents/analytic tools.

This work was supported by Georgetown University Partners in Research program (Deborah Wilson and Anthony Herman). K.C. was supported by National Institute of Mental Health R21MH118749 and supplies. S.A. was supported by the Saudi Arabian government, Qassim University scholarship program. M.M. was supported by NIH T32 NS041218. The work performed by the Postmortem Brain Core was supported in part by the IDeA Program of the National Institute of General Medical Sciences of the National Institutes of Health, Center for Psychiatric Neuroscience-COBRE (P30GM103328) and National Institute of Mental Health R01 MH67996. We apologize to investigators whose excellent work was not directly cited. We thank the Department of Comparative Medicine for outstanding veterinary support; Adam Caccavano for software development and sharing and assistance with images; the families for the invaluable contributions of consenting to donate brain tissue and be interviewed; Cuyahoga County Medical Examiner's Office (Cleveland, Ohio) for support; and Drs. James Overholser, George Jurjus, and Lesa Dieter for the psychiatric assessments; and Timothy M. De Jong and Lisa Konick for acquiring written consent and collecting tissues.

The authors declare no competing financial interests.

Correspondence should be addressed to Katherine Conant at kec84@georgetown.edu.

https://doi.org/10.1523/JNEUROSCI.2387-19.2020

Copyright $\odot 2020$ the authors

\section{Introduction}

Major depressive disorder (MDD) is a common psychiatric illness afflicting $7 \%-12 \%$ of men and $20 \%-25 \%$ of women (Belmaker and Agam, 2008). Untreated MDD increases one's risk for substance abuse, dementia, and suicide. Emerging evidence suggests that MDD is also associated with a reduction in overall excitatory to inhibitory (E/I) balance (for review, see Thompson et al., 2015; Page and Coutellier, 2019). For example, depressed patients show hypoactivity in the PFC (Fales et al., 2009). Similarly, animal models related to depression show atrophy of distal pyramidal dendrites in the PFC, loss of dendritic spines, and reduced expression of the AMPA glutamate receptor subunit GluA1 (Liston et al., 2006; Radley et al., 2008). In the hippocampus, chronically stressed animals also show atrophy of distal apical dendritic branches in excitatory neurons, and decreases in dendritic spine size and number (Magarinos and 
McEwen, 1995; Pawlak et al., 2005). A layer-specific decrease in GluA1 and postsynaptic density-95 (PSD-95) expression has also been described previously (Kallarackal et al., 2013). A better understanding of how E/I balance is altered in depression, and how it may be restored by therapy, could lead to the development of improved therapeutics.

Levels of matrix metalloproteases (MMPs), a family of zincdependent endopeptidases that play a role in adaptive and maladaptive learning and memory (Nagy et al., 2006; Meighan et al., 2007; Conant et al., 2010; Smith et al., 2014), may be increased by commonly prescribed monoamine reuptake inhibitors, and their activity may also enhance E/I balance (Alaiyed et al., 2019). For example, monoamines stimulate G-protein coupled receptors that increase MMP expression, including the D1 dopamine receptor and the 5-HT-7 serotonin receptor (Li et al., 2016; Bijata et al., 2017). In terms of E/I balance, MMPs have been shown to stimulate dendritic arborization of pyramidal neurons as well as dendritic spine formation and expansion (Wang et al., 2008; Allen et al., 2016). In addition, through cleavage of perineuronal net (PNN) components, MMPs may reduce glutamate input to, and increase membrane capacitance of, parvalbumin (PV)-expressing GABA-releasing interneurons, thereby disinhibiting pyramidal neurons to indirectly enhance E/I balance (Frischknecht et al., 2009; Tewari et al., 2018).

Herein we evaluate the possibility that MMP-9, which is released from neurons in a neuronal activity-dependent manner and is critical for learning and memory in varied brain regions (Nagy et al., 2006; Ganguly et al., 2013; Smith et al., 2014), mediates antidepressant-associated increases in E/I balance. We test this in both conventionally housed C57BL/6J mice and C57BL/6J mice subjected to a corticosterone (CORT)-induced stress model of depression (David et al., 2009). The CORT model induces an anxiety and depressive phenotype in C57BL/6 mice, a strain that is resistant to several stress protocols (David et al., 2009). CORTinduced phenotypic changes in C57BL/6 mice include abnormalities in novelty-suppressed feeding and open field performance (David et al., 2009). Our focus is MMP-dependent effects on dendritic arbor in excitatory pyramidal neurons, as well as PSD95 levels in hippocampal lysates, both of which have the potential to influence E/I balance. We also examine effectors of E/I balance that instead involve MMP-dependent effects on inhibitory GABA-releasing interneurons. These include the expression of PNN components, the power of $\gamma$ oscillations, the abundance of ex vivo sharp wave ripples (SWRs), and working memory performance. The power of $\gamma$ oscillations contributes to working memory capacity and is reduced in MDD (Yamamoto et al., 2014; Arikan et al., 2018). Moreover, $\gamma$ power is normalized with symptomatic remission (Arikan et al., 2018). To further explore the relevance of our findings to MDD, we also examined MMP-9 and tissue inhibitor of metalloproteinase-1 levels in postmortem PFC samples from control, MDD, and antidepressant-treated subjects with MDD. In all studies, we focused on the hippocampus and/or PFC, two regions implicated in MDD (MacQueen and Frodl, 2011).

\section{Materials and Methods}

\section{Chemicals and reagents}

( \pm )-Isoproterenol hydrochloride was purchased from U.S. Pharmacopeia (USP catalog \#35100). ( \pm )-Venlafaxine (VFX) and carbamoylcholine chloride (carbachol) were purchased Sigma Millipore (catalog \#PHR1736 and \#C4382, respectively). ( \pm )-Norepinephrine (+)-bitartrate salt was purchased from Sigma Millipore (catalog \#A0937), and sotalol hydrochloride was purchased from Tocris Bioscience (catalog \#0952).

\section{Cell culture and treatments}

Primary hippocampal neuronal cells were prepared from WT postnatal day $0-1$ mouse pups. The hippocampi were dissected after the removal of the meninges and dissociated into a single-cell suspension with titration following $15 \mathrm{~min}$ incubation with $0.25 \%$ trypsin (Beaudoin et al., 2012). Neuronal cells were plated on $18 \mathrm{~mm}$ sterilized glass coverslips in a 12-well plate at $\sim 90,000$ cells concentration. Cultured cells were maintained in neural basal media supplemented with B27, glutamine, and penicillin/streptomycin antibiotic and incubated at $37^{\circ} \mathrm{C}$ in a humidified $5 \% \mathrm{CO}_{2}$-containing atmosphere for $18 \mathrm{DIV}$. Treatment was started at 14-18 DIV. Cells were treated with isoproterenol $(30 \mu \mathrm{M})$, norepinephrine $(25 \mu \mathrm{M})$, or sotalol $(20 \mu \mathrm{M})$ for $24 \mathrm{~h}$. Neuronal culture supernatants were concentrated 10-fold (VWR filter concentrators, catalog \#\#82031344) and saved for ELISA.

\section{Mice}

Animals used for experiments were sex-matched as indicated in Results, and $\sim 4$ weeks old at the start of treatment. Because CORT may have differential effects as a function of sex, with females less sensitive to some endpoints and males to others (Mekiri et al., 2017; Berger et al., 2019; Yohn et al., 2019), results are reported for single-sex (male) comparisons. Strains were C57BL/6J (The Jackson Laboratory, RRID: IMSR JAX:000664) or MMP-9 homozygous null mice (The Jackson Laboratory, RRID: IMSR_JAX:007084) that had been backcrossed to C57BL/6J mice for at least five generations. Experimental groups were arbitrarily assigned to treatment groups. Mice were housed 4 or 5 per cage. Food and water were provided ad libitum. Experiments were performed in accordance with National Institutes of Health guidelines and institutionally approved protocols (2016-1117 and 2018-0037). Cages were supplied with balconies and nesting materials for enrichment.

\section{CORT and VFX treatment}

Mice that received saline or VFX were treated for 2 weeks with a daily intraperitoneal injection of sterile saline or $30 \mathrm{mg} / \mathrm{kg}$ VFX in sterile saline (200 $\mu \mathrm{l}$ total volume). For the depression model used herein (David et al., 2009), mice were treated with CORT ( $35 \mu \mathrm{g} / \mathrm{ml}$ equivalent to $5 \mathrm{mg} /$ $\mathrm{kg}$ /day) dissolved in $0.45 \% \beta$-cyclodextrin for 6 weeks and added to their drinking water. Because CORT may have differential effects as a function of sex, results are reported for single-sex comparisons (Mekiri et al., 2017; Berger et al., 2019; Yohn et al., 2019). Control mice received $0.45 \% \beta$-cyclodextrin in their drinking water. For experiments that also evaluated the effects of VFX in this model, during the last 2 weeks of the protocol mice were assigned to groups in which they received intraperitoneal VFX $(30 \mathrm{mg} / \mathrm{kg})$ or an equal volume of saline daily. To minimize animal distress, injections were delivered by trained veterinary technicians and the delivery site alternated from right to left each day.

\section{T-maze and elevated plus maze (EPM) testing}

EPM testing was performed using a standard apparatus and an ANYmaze video tracking as previously described (Allen et al., 2016). Working memory was assessed by T-maze testing (Shoji et al., 2012). The T-shaped apparatus was placed with the stem of the $\mathrm{T}$ proximal to the experimenter. Testing was begun with the distal right and left drop down doors raised. Unlike the reward version of this test, no habituation to the maze is used, as it is the novelty of the maze that tests spontaneous alternation. Each mouse was placed in the start arm with the door lowered, and following removal of this door the animal was allowed to freely choose to enter either the right or left arm. After the mouse chose a goal $\mathrm{arm}$, the door was lowered to confine the mouse in that arm for $30 \mathrm{~s}$. The mouse was then removed gently, placed into a rest cage from which the maze was not visible, the maze was cleaned, and the dropdown doors to the distal right and left arms raised. At that point, the same mouse was again placed in the start arm and the test repeated. This was done sequentially for each mouse in the cohort, and then sequentially repeated. Choices were recorded for each trial as a 1 (alternation) or 0 (no alternation). 
SWR and $\gamma$ recordings

Hippocampal slices and local field potential (LFP) recordings of $\gamma$-frequency oscillations and SWRs were prepared as previously described (Alaiyed et al., 2019). Briefly, following the last day of VFX or saline injection, mice were anesthetized by isoflurane inhalation. After confirming unconsciousness by an inability to respond to a strong paw pinching, animals were rapidly decapitated and the whole brain extracted and chilled in cold $\left(0^{\circ} \mathrm{C}\right)$ sucrose-based cutting aCSF containing the following (in $\mathrm{mM}$ ): 252 sucrose, $3 \mathrm{KCl}, 2 \mathrm{CaCl}_{2}, 2 \mathrm{MgSO}_{4}, 1.25$ $\mathrm{NaH}_{2} \mathrm{PO}_{4}, 26 \mathrm{NaHCO}_{3}, 10$ dextrose, and bubbled with $95 \% \mathrm{O}_{2} / 5 \% \mathrm{CO}_{2}$. Hippocampal slices ( $490 \mu \mathrm{m}$ thick) were cut in horizontal sections from dorsal to ventral locations with a vibratome (Leica Microsystems, VT1000S). To minimize potential dorsal to ventral activity differences, we recorded from the 6-8 slices obtained when cutting began at the dorsal most aspect of the brain. Slices were transferred into a chamber filled with aCSF containing the following (in $\mathrm{mM}$ ): $132 \mathrm{NaCl}, 3 \mathrm{KCl}, 2 \mathrm{CaCl}_{2}$, $2 \mathrm{MgSO}_{4}, 1.25 \mathrm{NaH}_{2} \mathrm{PO}_{4}, 26 \mathrm{NaHCO}_{3}, 10$ dextrose, and saturated with $95 \% \mathrm{O}_{2} / 5 \% \mathrm{CO}_{2}$ at $32^{\circ} \mathrm{C}$. At $120 \mathrm{~min}$, heating was discontinued and the aCSF allowed to gradually cool to room temperature $\left(\sim 25^{\circ} \mathrm{C}\right)$. Slices were incubated for at least $180 \mathrm{~min}$ in total before being moved to the recording chamber. For LFP recordings of $\gamma$-frequency oscillations and SWR, low-resistance glass microelectrodes ( $\sim 150 \mathrm{~K}$ tip resistance) were used. Electrodes were filled with $1 \mathrm{M} \mathrm{NaCl}$. Recordings were performed in CA1 stratum oriens proximal to CA2. The aCSF fluid was switched to aCSF-containing carbachol at $40 \mu \mathrm{M}$ for recording of carbachol-stimulated $\gamma$ oscillations. Local field analyses were performed with a previously described custom MATLAB algorithm, available on request (Alaiyed et al., 2019).

Preparation of brain lysates and fixed tissue

Following treatment and death, brains were bisected into right and left hemi-brains. Homogenates from hippocampal and cortical tissue were prepared by lysis in immunoprecipitate buffer [50 mm Tris, $\mathrm{pH} 7.5,150$ $\mathrm{mm} \mathrm{NaCl}, 0.1 \%$ SDS, $1 \%$ octylphenoxypoly (ethyleneoxy) ethanol, branched, and $1 \times$ protease and phosphatase cocktail (Thermo Fisher Scientific, 1861281)]. Lysates were sonicated for $10 \mathrm{~s}$, placed on ice for $20 \mathrm{~min}$, and centrifuged $15 \mathrm{~min}$ at $14,000 \mathrm{rpm}$ at $4^{\circ} \mathrm{C}$. Lysate supernatants were saved for protein analyses. For immunohistochemistry, hemibrains were rapidly fixed in chilled $4 \%$ PFA overnight at $4^{\circ} \mathrm{C}$ before paraffin embedding and thin slice preparation $(15 \mu \mathrm{m})$.

\section{ELISA and Western blot}

Pro-MMP9 protein concentrations in cell culture supernatants and tissue lysates were measured by ELISA, performed according to the manufacturer's instructions (Mouse Pro-MMP-9, R\&D Systems, catalog \#MMP900B). For immunoblotting, protein concentrations were determined using a BCA protein assay (Pierce Biotechnology), and equal amounts of protein were loaded in each lane. Samples $(50 \mu \mathrm{g}$ total protein) were immersed in Laemmli sample buffer (Bio-Rad, catalog \#1610737) containing $5 \% \beta$-mercaptoethanol, and boiled for $5 \mathrm{~min}$ at $95^{\circ} \mathrm{C}$. Samples were subsequently separated by electrophoresis on precast gels (4\%-20\% mini protein TGX gels, Bio-Rad, catalog \#456-1094) and transferred to nitrocellulose membranes (Trans-Blot Turbo Transfer, BioRad, catalog \#1704159). Membranes were probed with primary and secondary antibodies, and bands visualized by chemiluminescence as previously described (Alaiyed et al., 2019). Primary antibodies used were mouse anti-brevican (BCAN, 1:1000, 610894, BD Transduction Laboratories), mouse anti-PSD-95 (1:1000, CP35, Millipore), and mouse anti-GAPDH (1:5000, MAB374, Millipore).

\section{Golgi staining and analysis}

Golgi staining was performed with the FD Rapid Golgi Stain Kit (FD NeuroTechnologies, catalog \#PK401) according to the manufacturer's instructions. After death, murine brains were quickly removed, rinsed in double-distilled water, and immersed in the impregnation solution.
Brains were stored in this solution at room temperature for 2 weeks in the dark. Tissue was subsequently transferred to solution $\mathrm{C}$ and stored at room temperature for 1 week. Brains were sectioned $(150 \mu \mathrm{m})$ with a vibratome (Leica Microsystems, VT1000S). Each section was then transferred and mounted on gelatin-coated microscope slides and allowed to dry naturally at room temperature in the dark. After rinsing in doubledistilled water 2 times, 4 min each, slides were put into the manufacturer-recommended mixture of staining solutions for $10 \mathrm{~min}$ and then rinsed. Sections were dehydrated in 50\%, 75\%, 95\%, 100\% ethanol, and tissue-clear for $4 \mathrm{~min}$ each and then mounted with Hydromount (National Diagnostics, HS-106). Visualization of arbors and quantification was subsequently performed as described previously (Allen et al., 2016). Four or five mice were used per group, and one or two slides were analyzed per mouse (total 9 or 10 slides/mouse). Slide identity was coded and quantification of arbors was performed by an investigator blind to cohort.

\section{Immunostaining}

Immunostaining was performed as described previously (Alaiyed et al., 2019). Briefly, following fixation in $4 \%$ PFA, brains were paraffin-embedded and sectioned $(15 \mu \mathrm{m})$. Sections were washed 2 or 3 times with $1 \times$ PBS, then permeabilized with $1 \times$ PBS containing $0.1 \%$ Triton X-100, blocked with $10 \%$ normal goat serum, and incubated with anti-PV (1:500, Sigma Millipore, P3088) overnight at $4^{\circ} \mathrm{C}$. Following subsequent washes and incubation with a fluorescent secondary antibody for PV immunostaining and fluorescein-labeled Wisteria floribunda lectin (WFA) (1:1000, Vector Laboratories, FL-1351) for $2 \mathrm{~h}$ at room temperature, sections were washed several times with $1 \times \mathrm{PBS}$, counterstained with DAPI, and mounted with Hydromount (National Diagnostics, HS-106) before being allowed to dry several days at $4^{\circ} \mathrm{C}$. For PV and PNN cell quantification, images were acquired using a Leica SP8 laser scanning confocal microscope with an oil immersion, 209 objective with $0.40 \mathrm{nu}-$ merical aperture. Laser intensity, gain, and pinhole settings were kept constant for all samples. Images were taken through a $z$ plane $(8.5 \mu)$ within the center of the tissue section, containing 20 stacks $(0.4 \mu /$ stack $)$ from the dorsal hippocampus. Quantification of PV numbers with and without an associated PNN was counted for each image that was acquired from ROIs using 8-10 mice per group, and two or three slides per mouse. For PNN intensity, a semiautomated analysis, PIPSQUEAK macro plugin, was used described by Slaker et al. (2015). This macro uses an ROI protocol for double-labeled cell-based quantification. Following background subtraction within an appropriate radius, WFA or PV cells were identified based on threshold limit requirements. ROIs were identified around WFA-labeled PV-expressing cells, and the mean intensity was measured. Data points represent averages for ROIs from one or two images from each of animal.

\section{Human tissue analyses}

The Institutional Review Boards of the University of Mississippi Medical Center (Jackson, MS) and the University Hospitals Cleveland Medical Center (Cleveland) approved all procedures of tissue collection and retrospective psychiatric assessment. Informed consent was obtained from legally defined next of kin for tissue collection, interviews, and medical records. A master-level social worker administered the Structured Clinical Interview for DSM-IV Axis I Disorders to informants with knowledge of the subjects. A board-certified clinical psychologist and a board-certified psychiatrist independently reviewed the scoring of the diagnostic interview, medical records, and the medical examiner's report. Psychopathology of the subjects, or the lack thereof in control subjects, was determined in consensus with the clinical psychologist, the psychiatrist, and the social worker. Subjects meeting DSM-IV criteria for MDD, or controls without a psychiatric diagnosis were selected for this study. Subjects with any neuropathological or neurologic disorders were excluded. The medical examiner's office assessed the presence of psychotropic medications and substances of abuse in blood and urine.

Samples of postmortem brain tissue were acquired at autopsy at the Cuyahoga County Medical Examiner's Office (Cleveland). The medical examiner determined the cause of death. Gray matter tissues from the 
A

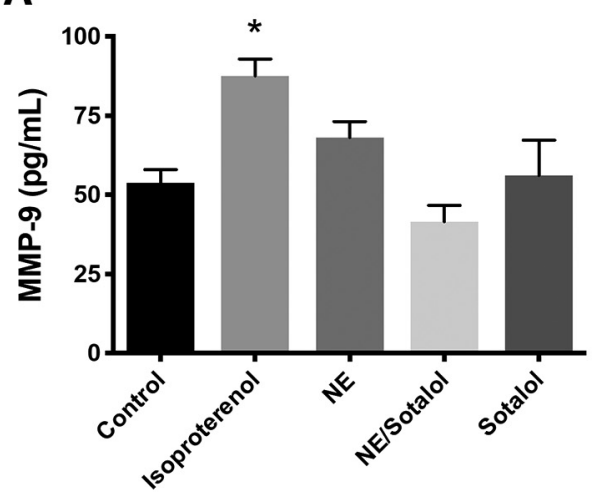

B

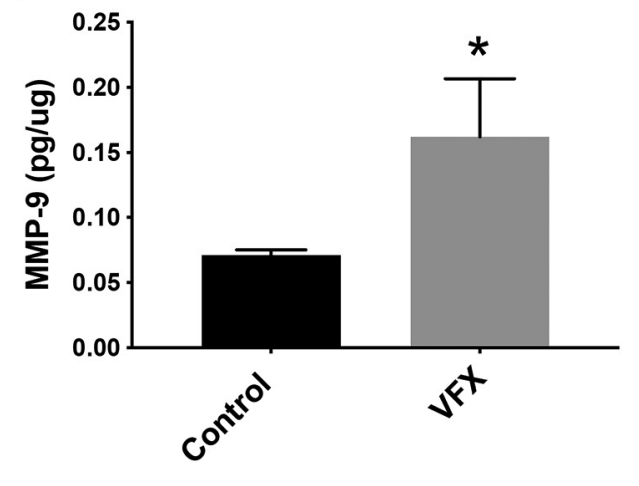

Figure 1. $\quad \beta$-adrenergic receptor activation increases MMP-9 release from cultured neurons, and MMP-9 levels are increased in brain lysates from VFX-treated animals. $\boldsymbol{A}$, The nonselective $\beta$-adrenergic agonist isoproterenol stimulated a significant increase in the release of MMP-9 from cultures of murine hippocampal neurons ( $n=5$ or 6 biological replicates, $p=0.0013$, oneway ANOVA with Tukey's post hoc testing). In addition, while the difference between isoproterenol and norepinephrine was not significant, the difference between isoproterenol and all other groups did reach significance. $\boldsymbol{B}$, MMP-9 levels in cortical lysates from animals that were treated for 2 weeks with saline or VFX. VFX was associated with a significant increase in cortical MMP9 (pg/ $\mu \mathrm{g}$ total tissue, $n=4$ or 5 mice per group, $p=0.044$, Student's $t$ test). ${ }^{*} p<0.05$.

frontal pole (Brodmann's area 10) were dissected and rapidly frozen in 2 -methylbutane cooled by dry ice, and subsequently buried in powdered dry ice before storage at $-80^{\circ} \mathrm{C}$. Samples were segregated into three groups, age- and sex-matched, and coded so that research staff were unaware of diagnoses. The groups $(n=12-15)$ included psychiatrically normal control subjects, subjects with MDD but had not been treated with an antidepressant medication in the weeks before death, and subjects with MDD that were treated with an antidepressant medication having a proximal effect on the monoamine neurotransmitter system (paroxetine $n=1$; fluoxetine $n=3$; VFX $n=2$; amitriptyline $n=2$; amitriptyline + mirtazapine $n=1$; sertraline $n=1$; trazodone $n=1$; and tramadol $n=1$ ). Paroxetine, fluoxetine, trazodone, sertraline, and VFX inhibit reuptake of serotonin and/or norepinephrine, neurotransmitters that may increase in vitro MMP-9 release (Bijata et al., 2017). Mirtazapine increases brain levels of dopamine and norepinephrine (Millan et al., 2000). Amitriptyline is converted to nortriptyline, which is a potent and selective norepinephrine reuptake inhibitor, and tramadol is an serotonin and norepinephrine reuptake inhibitor in addition to a opioid agonist (Barber, 2011). Antidepressant treatment was confirmed by postmortem toxicology in MDD subjects, and these subjects also had an active prescription in the last month of life. The mean age, age range, and postmortem delay \pm SD for each group were as follows: Control (age $51 \pm 15.1 \mathrm{yr}$, postmortem delay $22.2 \pm 7.5 \mathrm{~h}$ ); MDD (age $50 \pm 15.7$ yr, range 28-83 yr; postmortem delay $22 \pm 6.5 \mathrm{~h}$ ); MDD plus antidepressant (age $52 \pm 16.5 \mathrm{yr}$, postmortem delay $23.6 \pm 8.3 \mathrm{~h}$ ). Brain lysates were prepared according to established methods (St Hillaire et al., 2005), and ELISA for MMP-9 and TIMP-1 was performed using commercially available kits (R\&D Systems). Data were analyzed and corrected for total protein levels before sending it to coinvestigators to break the diagnostic code on samples.

Experimental design and statistical analyses

Sample size was based on experience indicating that 4-8 animals per group are typically necessary to observe physiologically relevant changes in MMP levels and additional biochemical endpoints (Alaiyed et al., 2019). Experiments focused on PNN intensity and population recordings were set up to use 4-6 animals and several slices from each as indicated in Materials and Methods. Data were entered into a GraphPad Prism 8.0 program, and statistical analysis was performed using Student's unpaired $t$ test for two group comparisons or ANOVA, with post hoc analyses as indicated, for comparisons of more than two groups. Multiple comparisons controlled for and within- and between- subject comparisons as indicated in Results for human subject data. Significance was set at $p<0.05$, and ROUT testing was performed to identify outliers. For parametric analyses, normality was tested with Shapiro-Wilk or Kolmogorov-Smirnov testing. Statistical data, including degrees of freedom, are included in the results section.

\section{Results}

$\beta$-Adrenergic receptor activation increases MMP-9 release from cultured neurons and MMP-9 levels are increased in brain lysates from VFX-treated animals

Previous studies have demonstrated that $5 \mathrm{HT}-7$ serotonin receptor activation, which is linked to $G \gamma_{s}$ stimulation, increases MMP-9 activity in cultured neurons (Bijata et al., 2017). Higher doses of VFX, as used in this study, can also increase extracellular levels of norepinephrine. We therefore examined agonists of $\mathrm{G} \gamma_{\mathrm{s}}$-coupled norepinephrine receptors for their ability to stimulate an increase in MMP-9 release from cultured murine hippocampal neurons. As shown in Figure $1 A$, the nonselective $\beta$-adrenergic agonist isoproterenol stimulated a significant increase in the release of MMP-9 from cultured murine hippocampal neurons $\left(n=5\right.$ or 6 biological replicates, $F_{(4,24)}=6.275$, $p=0.0013$, one-way ANOVA with Tukey's post hoc testing). While the difference between isoproterenol and norepinephrine was not significant, the difference between isoproterenol and all other groups did reach significance.

Because serotonin and norepinephrine reuptake inhibitors will indirectly activate monoamine receptor subtypes linked to varied downstream pathways, it was important to also assess MMP-9 levels in animals treated with monoamine reuptake inhibitors. We previously demonstrated that VFX increases levels of MMP-9 in murine hippocampus (Alaiyed et al., 2019). Since some of the experiments in the present study are instead focused on the cortex, in Figure 1B, we examined MMP-9 in sensorimotor cortical lysates from animals that were treated for 2 weeks with saline or the VFX. Similar to what was observed in the hippocampus, VFX was associated with a significant increase in cortical expression of MMP-9 (pg/ $\mu$ g total tissue, $n=4$ mice per group, $t_{(6)}=2.03, p=0.044$, Student's $t$ test).

\section{VFX stimulates increased dendritic arbor and PSD-95 levels in WT but not MMP-9-null mice}

MMPs have been shown to enhance dendritic arborization and spine formation in varied settings (Wang et al., 2008; Verslegers et al., 2015; Allen et al., 2016). Potential mechanisms include their ability to activate proneurotrophins (Lee et al., 2001), their ability to generate integrin-binding ligands (Lonskaya et al., 2013), and their ability to modulate extracellular matrix in a manner that may be permissive for spine growth (Fawcett et al., 
A
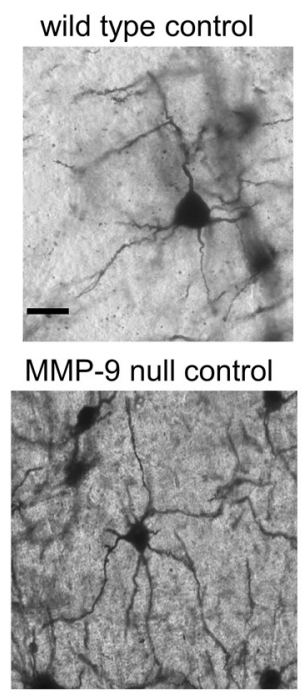

wild type VFX
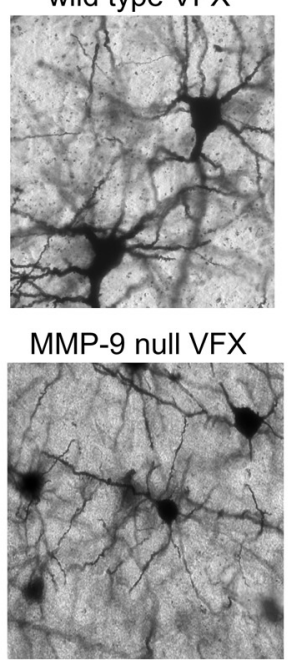

B

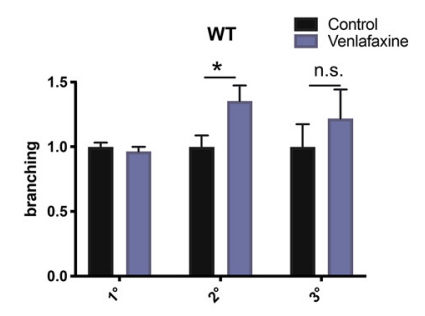

C

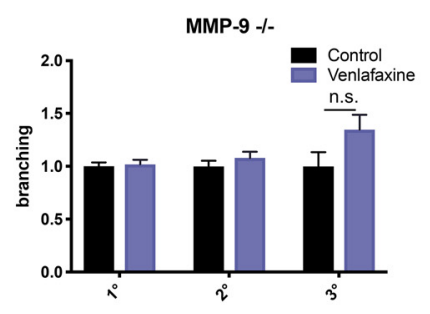

D

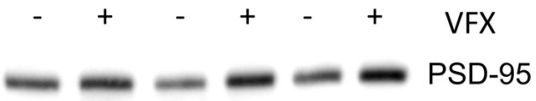

- - - - $-\mathrm{GAPDH}$

E Wild type

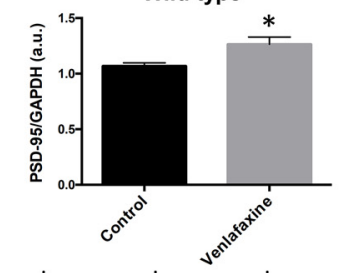

$\mathbf{F}$

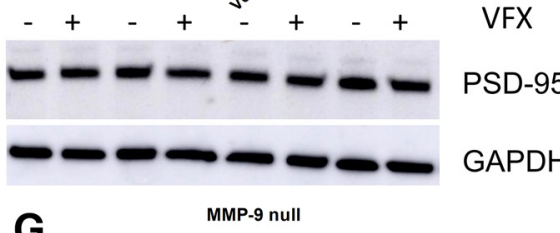

G

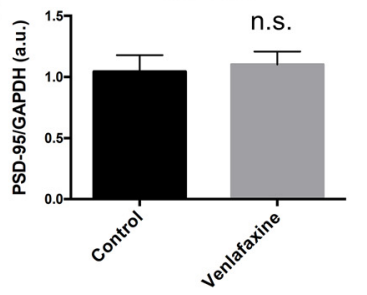

Figure 2. VFX stimulates increased dendritic arbor and PSD-95 levels in WT but not MMP-9-null mice. $A$, Representative images from Golgi-stained cortex for mouse strains and treatment groups. $\boldsymbol{B}, \boldsymbol{C}$, Normalized branching data for primary, secondary, and tertiary processes. D-G, Results from PSD-95 quantification in hippocampal lysates. $\boldsymbol{B}, \boldsymbol{C}$, VFX significantly increases the number of secondary dendrites in WT but not MMP-9-null mice ( $n=39-45$ neurons from 6-8 mice per group, WT control vs VFX: $p=0.0176$, Student's $t$ test; MMP-9-null control vs VFX: not significant). $\boldsymbol{D}-\mathbf{G}$, VFX significantly increased PSD-95 levels in WT mice ( $p=0.0388$, Student's $t$ test). There was, however, no significant difference in PSD-95 levels between the saline and VFX-treated MMP-9 KOs. Scale bar, $20 \mu \mathrm{m} .{ }^{*} p<0.05$, n.s., non significant.

2019). To determine whether VFX had the potential to enhance dendritic arbor and spine formation in conventionally housed mice, we treated animals for $14 \mathrm{~d}$ with $30 \mathrm{mg} / \mathrm{kg}$ VFX and subsequently performed Golgi analyses to examine arborization and Western blots to quantify levels of PSD-95, which is typically increased in the setting of dendritic spine expansion and/or increased formation. Because the high density of dendritic arbors in hippocampus hinders quantification, arborization was quantified in the sensorimotor cortex, which receives abundant serotonergic and noradrenergic inputs and demonstrates pathology in MDD (Kang et al., 2018; Kropf et al., 2019). Representative images are shown in Figure $2 A$, and quantitation of branching data for primary, secondary, and tertiary processes is shown in Figure $2 B, C$. Results from PSD-95 quantification in hippocampal lysates are shown in Figure $2 D-G$. As shown in Figure $2 B, C$, VFX significantly increases the number of secondary dendrites in WT but not MMP-9-null mice $(n=37-45$ neurons from 6-8 mice per group, WT control vs VFX: $t_{(94)}=2.416, p=0.0176$, Student's $t$ test; MMP-9-null control vs VFX: not significant, $t_{(73)}=1.01, p=0.32$, Student's $t$ test). And while tertiary dendritic branching appears increased by VFX in both the WT and MMP-9 KOs, the difference in tertiary branches for saline and VFX was not significant in either group, possibly due to a lower number of tertiary compared with secondary processes. Normalized values are shown since the WT \pm VFX and the MMP-9-null \pm VFX groups were stained at different times. As shown in Figure 2D-G, VFX significantly increased PSD95 levels in WT mice $\left(n=6, t_{(7)}=2.54, p=0.0388\right.$, Student's $t$ test). There was, however, no significant difference in PSD-95 levels between the saline and VFX-treated MMP-9 KOs $\left(t_{(6)}=\right.$ $0.4, p=0.659$, Student's $t$ test).
VFX increases BCAN cleavage in WT but not in MMP-9-null mice

While select changes in pyramidal cell structure can enhance E/I balance, reduced inhibitory input to pyramidal cells can do the same. PV-expressing interneurons are among the GABA-releasing neuronal subtypes that can inhibit cortical and hippocampal pyramidal cells. PV-expressing interneurons are also the predominant neuronal population that is surrounded by the PNN, a dense form of extracellular matrix that can be cleaved by MMPs. PNNs provide PV neurons with decreased membrane capacitance and also localize glutamate input to synaptic contacts, thus facilitating the release of GABA from PV interneurons and thereby inhibiting pyramidal neuron activity (Frischknecht et al., 2009; Tewari et al., 2018). Disruption of the PNN can instead reduce PV-mediated pyramidal cell inhibition (Hayani et al., 2018).

To examine the potential for VFX to upregulate cleavage of specific PNN components, we examined the integrity of BCAN in saline and VFX-treated animals. We also examined basal and VFX-stimulated BCAN levels and cleavage in WT compared with MMP-9-null animals. We focused on BCAN because it is a critical PNN component with respect to PV interneuron function (Favuzzi et al., 2017). In Figure $3 A-C$, we show full-length and cleaved BCAN (80 kDa) in control and MMP-9-null mice. Representative Western blot results are shown in Figure $3 A$, and densitometric results from 4 or 5 mice per group are shown in Figure $3 B, C$. It can be appreciated that, while full-length BCAN does not significantly differ between WT and MMP-9-null mice $\left(t_{(7)}=1.3, p=0.24\right.$, Student's $t$ test), basal BCAN cleavage is significantly reduced in MMP-9-null animals $\left(t_{(7)}=6.387, p=\right.$ 


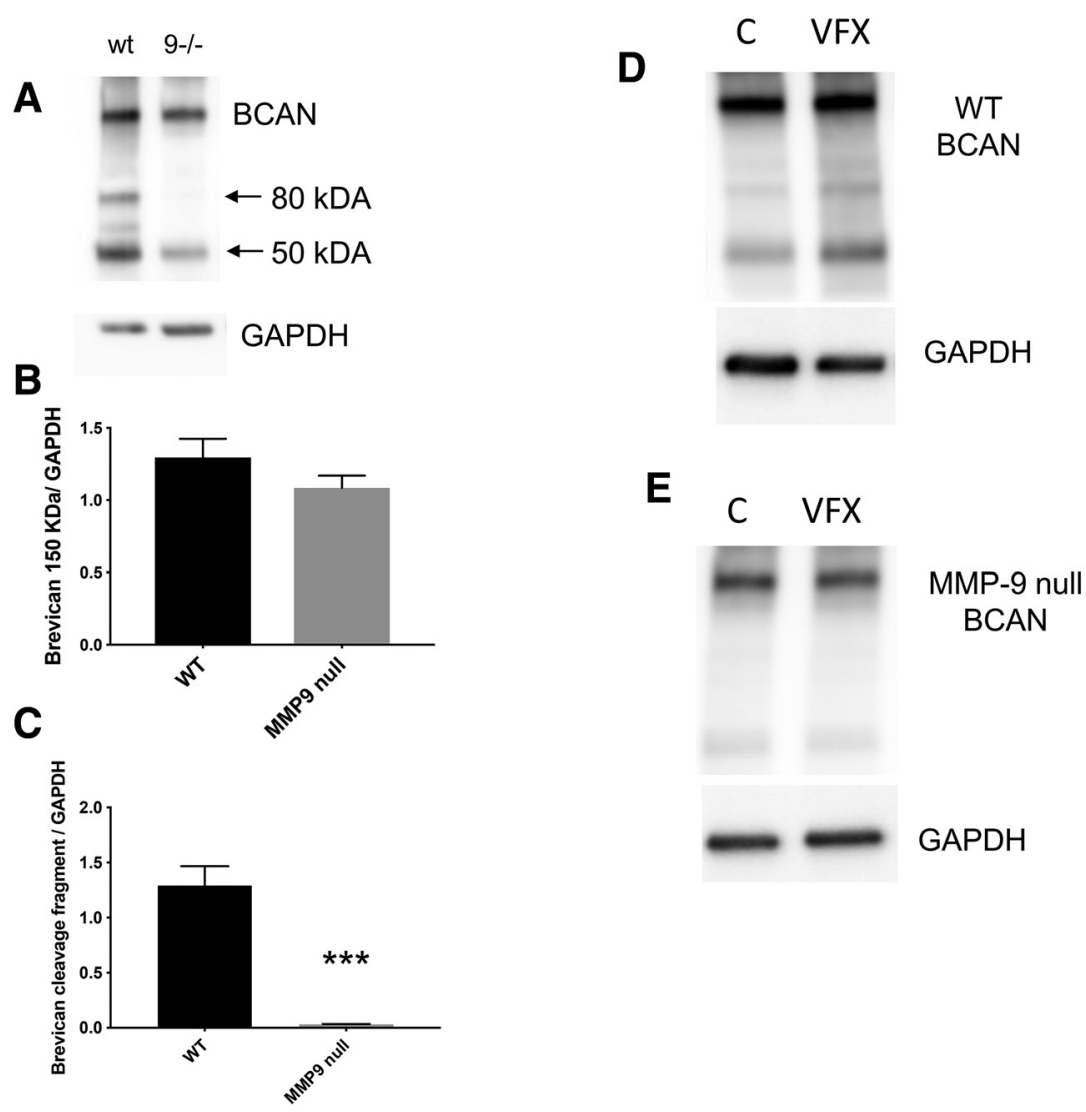

Figure 3. VFX increases BCAN cleavage in WT but not in MMP-9-null mice. $A$-C, Full-length and cleaved BCAN $(80 \mathrm{kDa})$ in control and MMP-9-null mice. $\boldsymbol{A}$, Representative Western blot results. $B, C$, Densitometric results from 4 or 5 mice per group. It can be appreciated that, while full-length BCAN does not significantly differ between WT and MMP-9-null mice $(p=0.24$, Student's $t$ test), basal BCAN cleavage is significantly reduced in MMP-9-null animals ( $p=0.0004$, Student's $t$ test). D-F, Full-length and cleaved BCAN in saline and VFX-treated WT and MMP9 KO mice. $\boldsymbol{D}, \boldsymbol{E}$, Representative Western blot results. $\boldsymbol{F}$, Densitometric analyses with subsequent quantification of the ratio of cleaved/full-length BCAN from $n=4$ or 5 mice per group. The difference between control and VFX in WT mice is significant ( $p=0.0278$, ANOVA with Sidak's multiple comparisons). In contrast, the difference between control and VFX in MMP-9-null animals is not significant ( $p>0.05$, ANOVA with Sidak's multiple comparisons). ${ }^{*} p<0.05,{ }^{* *} p<0.001$.

0.0004, Student's $t$ test). In Figure 3D-F, we show full-length and cleaved BCAN in saline and VFX-treated WT and MMP-9 KO mice. Representative Western blot results are shown in Figure $3 D, E$. Densitometric analyses with subsequent quantification of the ratio of cleaved/full-length BCAN from $n=4$ or 5 mice per group are shown in Figure $3 F$. The difference between control and VFX in WT mice is significant $\left(F_{(1,14)}=\right.$ 3.2; $p=0.0278$, ANOVA with Sidak's multiple comparisons). In contrast, the difference between control and VFX in MMP-9-null animals is not significant $(p=0.95$, ANOVA with Sidak's multiple comparisons).

\section{CORT increases BCAN and PNN levels}

While emerging evidence links stress and depression to a reduction in E/I balance, most studies have focused on changes in pyramidal cell structure and function that would be expected to diminish the ability of pyramidal cells to respond to glutamate. Few studies have explored the possibility that depression could be secondary to increased GABA-mediated inhibition. Since enhanced deposition of PNN components has the potential to increase PV interneuron-mediated inhibition (Page et al., 2019), we examined BCAN levels in mice exposed to a CORT-induced stress model of depression. We focused on BCAN expression in the hippocampus as this is a region rich in receptors for CORT. As shown in Figure $4 A, B, B C A N$ levels are increased in hippocampal lysates from CORT-exposed mice compared with controls ( $n=4$ mice per group, $t_{(6)}=2.5, p=0.0466$, Student's $t$ test). Western blot data are shown in Figure $4 A$, and the results from densitometric analyses are shown in Figure $4 B$. Because previous results suggest that MMP-9 is an important mediator of basal BCAN cleavage and VFX-stimulated PNN cleavage (Alaiyed et al., 2019), we also looked at ratios of MMP-9 and its inhibitor, TIMP-1, in hippocampal lysates from CORT-exposed animals treated with saline or VFX. As shown in Figure 4C, VFX significantly increased MMP-9/TIMP-1 levels over control ( $n=5$ or 6 mice per group, $F_{(2,13)}=6.98, p=0.0027$, one-way ANOVA with Tukey's multiple comparisons), whereas CORT alone had no significant effect compared with control ( $p=0.094$, one-way ANOVA with Tukey's multiple comparisons).

We have previously shown that VFX stimulates an MMP-9dependent decrease in PNN intensity in conventionally housed mice (Alaiyed et al., 2019). We here examined the effects of VFX on overall PNN levels in the CORT model. In Figure $4 D$, we show representative staining; whereas in Figure $4 E, F$, we show quantification of the PV/PNN ratio and PNN intensity in WT animals. VFX significantly increases the PNN/PV ratio in CORT-exposed animals (6-8 mice, $n=18$ - 26 slides, $F_{(2,56)}=3.53, p=0.0383$, ANOVA with Tukey's multiple comparisons) and reduces overall PNN fluorescence intensity $\left(F_{(2,63)}=12.48, p<0.0008\right.$, 


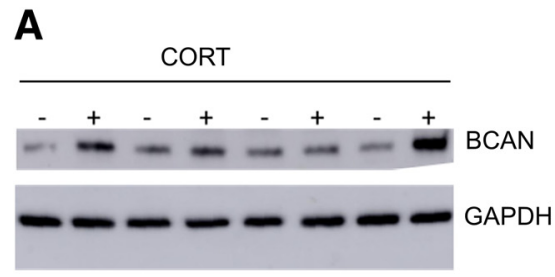

B
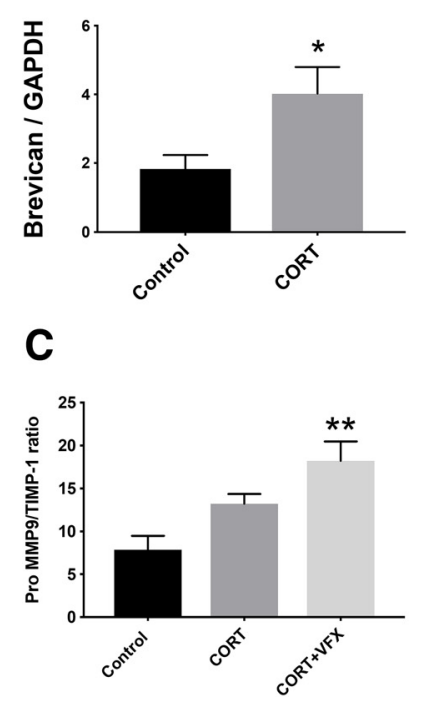

D

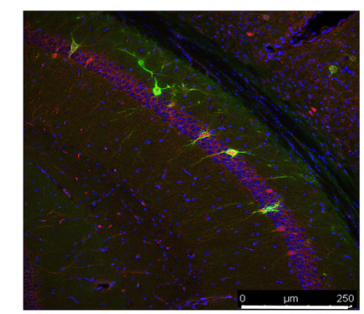

Control
E

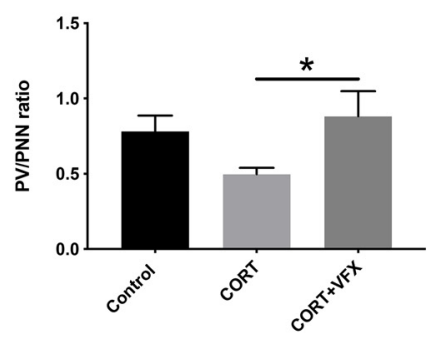

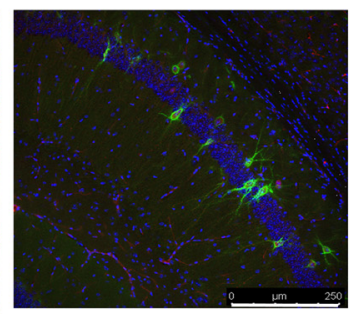

CORT

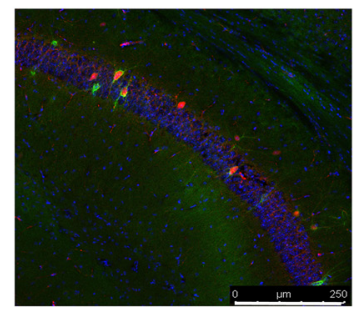

CORT+VFX
F

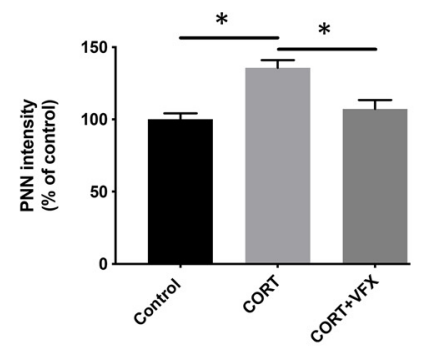

Figure 4. CORT increases BCAN and PNN levels. $A, B, B C A N$ levels in hippocampal lysates from control and CORT-exposed mice (Western blot and densitometry, respectively). BCAN levels are increased in hippocampal lysates from CORT-exposed mice compared with controls ( $n=4$ mice per group, $p=0.0466$, Student's $t$ test). $C$, Ratios of MMP-9 and its inhibitor, TIMP-1, in hippocampal lysates from CORT-exposed animals treated with saline or VFX. VFX significantly increased MMP-9/TIMP-1 levels over control ( $n=5$ or 6 mice per group, $p=0.0027$, one-way ANOVA with Tukey's multiple comparisons), whereas CORT alone had no significant effect compared with control ( $p=0.094$, one-way ANOVA with Tukey's multiple comparisons). D, Representative PNN staining for control, CRT, and CORT + VFX exposed WT animals. E, $F$, Quantification of the PV/PNN ratio and PNN intensity. VFX significantly increases the PNN/PV ratio in CORT-exposed animals (6-8 mice, $n=18-26$ slides, $p=0.0383$, ANOVA with Tukey's multiple comparisons) and reduces overall PNN fluorescence intensity ( $p<0.0008)$, consistent with a reduction in PNN levels. Scale bar, $250 \mu \mathrm{m} .{ }^{*} p<0.05,{ }^{* *} p<0.005$.

ANOVA with Tukey's multiple comparisons), consistent with a reduction in PNN levels.

VFX stimulates an MMP-9-dependent increase in the power of $\gamma$ oscillations in ex vivo hippocampal slices from CORTtreated animals

Since chronic CORT increased BCAN levels, and because the PNN is thought to facilitate the firing of PV interneurons and thus pyramidal cell inhibition, we next examined the power of $\gamma$ oscillations. $\gamma$ power is increased with PNN attenuation (Lensjo et al., 2017) and correlated with the magnitude of pyramidal cell mediated sharp waves. Importantly, $\gamma$ power is reduced in depression and animal models of depression (Khalid et al., 2016; Arikan et al., 2018). We previously demonstrated that VFX could increase carbachol-stimulated $\gamma$ power in hippocampal slices from conventionally housed, non-CORT-treated, animals (Alaiyed et al., 2019). Carbachol is used in slice studies of $\gamma$ to substitute for septal cholinergic input. As shown in Figure $5 A, B$, VFX also increases low and high $\gamma$ power in CORT-treated animals. The difference between CORT and CORT + VFX groups is significant (4-6 mice; $n=11-13$ slices; low $\gamma, p=0.026$, $F_{(2,34)}=4.9$; high $\gamma, p=0.016, F_{(2,34)}=5.2$; one-way ANOVA with Tukey's multiple comparisons), as is the difference between control and CORT groups (low $\gamma, p=0.041$, high $\gamma, p=0.038$, one-way ANOVA with Tukey's multiple comparisons). In contrast, the difference between controls and CORT + VFX groups is not significant. Shown in Figure $5 C$ are representative tracings from the three groups as indicated. We also evaluated the MMP9 dependence of VFX's effect on $\gamma$ power in the CORT model. As shown in Figure $5 D, E$, there is no significant difference between the CORT and CORT + VFX groups in MMP-9 KO animals ( 4 or 5 mice; $n=11$ or 12 slices; low $\gamma, F_{(2,33)}=0.569$, $p=0.54$; high $\gamma, F_{(2,33)}=0.68, p=0.49$; one-way ANOVA with Tukey's multiple comparisons). In Figure $5 F$, we show representative tracings from the $\mathrm{KO}$ animals. While the CORT and CORT + VFX groups show no difference in the MMP-9 KOs, it also appears that CORT has a lesser effect on $\gamma$ in the MMP-9-nulls compared with WT animals (control vs CORT conditions). One possibility is that MMP-9 could play a role in both CORT- and VFX-associated changes in $\gamma$. CORT-stimulated increases in MMP-9 might activate glia to enhance glial expression of PNN proteins, whereas VFX may increase MMP-9 in proximity to neurons to more than overcome effects of CORT in WT animals. Future studies will be necessary to address this possibility and others.

\section{VFX stimulates an MMP-9-dependent increase in SWR} abundance in ex vivo hippocampal slices from CORT-treated animals

While previous studies have linked MDD and animal models of the same to reduced $\gamma$ power, studies examining hippocampal SWR abundance in major depression or animal models are 
A
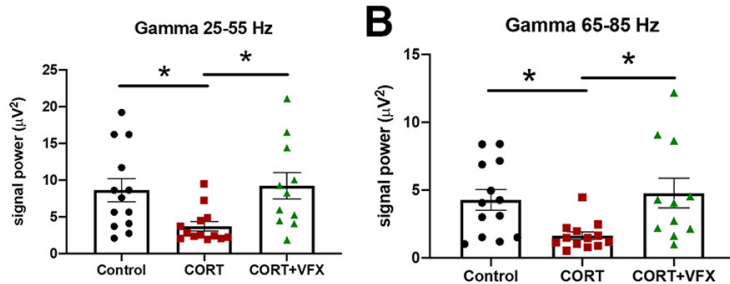

C

Control

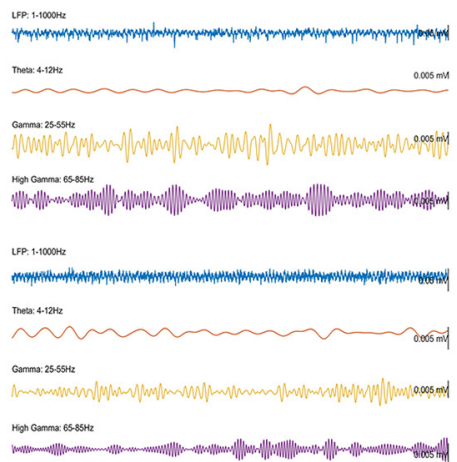

CORT+ VFX

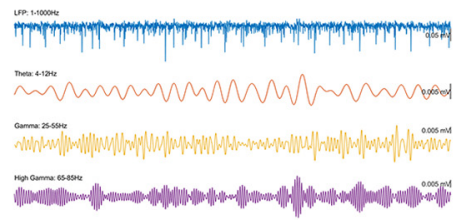

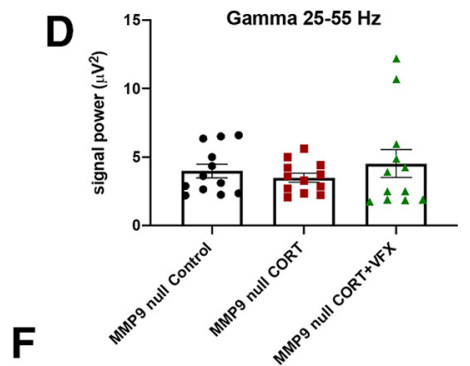

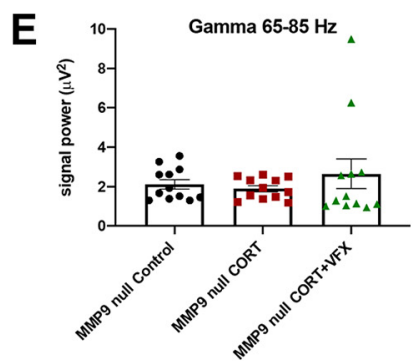

Control

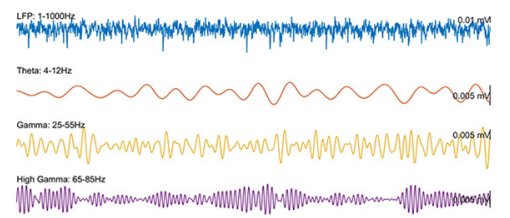

CORT

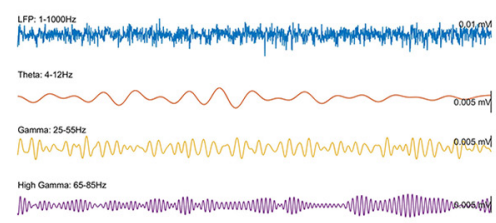

CORT+ VFX

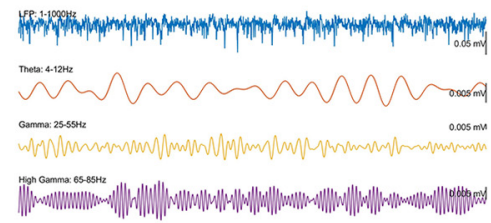

Figure 5. VFX stimulates an MMP-9-dependent increase in the power of $\gamma$ oscillations in ex vivo hippocampal slices from CORT-treated animals. $\boldsymbol{A}, \boldsymbol{B}$, Results from LFP recordings of carbachol-stimulated low and high $\gamma$ power in ex vivo hippocampal slices. VFX increases low and high $\gamma$ power in CORT-treated animals. The difference between CORT and CORT + VFX groups is significant (4-6 mice, $n=11-13$ slices, low $\gamma, p=0.026$, high $\gamma, p=0.016$, one-way ANOVA with Tukey's multiple comparisons), as is the difference between control and CORT groups (low $\gamma, p=0.041$, high $\gamma, p=0.038$, one-way ANOVA with Tukey's multiple comparisons). In contrast, the difference between controls and CORT + VFX groups is not significant. C, Representative tracings from the 3 groups as indicated. $\boldsymbol{D}, \boldsymbol{E}$, There is no significance difference between the CORT and CORT + VFX groups in MMP-9 K0 animals ( 4 or 5 mice, $n=11$ or 12 slices, low $\gamma, p=0.54$, high $\gamma, p=0.49$, one-way ANOVA with Tukey's multiple comparisons). $\boldsymbol{F}$, We show representative tracings from the K0 animals. ${ }^{*} p<0.05$.

lacking. Rapid sequential replay of hippocampal neuronal assemblies, initially activated in the same sequence during learning, occurs during SWRs and is important to memory consolidation (Ego-Stengel and Wilson, 2010). Importantly, declarative memory consolidation is reduced in patients with MDD (Nissen et al., 2010). We therefore examined SWR abundance in control and CORT-treated animals. As shown in Figure 6A, VFX increases SWR abundance in CORT-treated WT animals (4-6 mice; $n=$ 11-13 slices; $F_{(2,34)}=6.72, p=0.0025$; one-way ANOVA with Tukey's multiple comparisons). Representative traces for WT animals are shown in Figure 6B. In contrast, as shown in Figure $6 C$, VFX did not significantly increase SWR abundance in CORT-treated MMP-9-null mice (4 or 5 mice; $n=12$ slices; $F_{(2,33)}=2.74 p=0.9$; one-way ANOVA with Tukey's multiple comparisons). The difference between control and CORT groups and control and CORT/VFX groups was also nonsignificant ( $p=0.18$ and $p=0.08$, respectively, one-way ANOVA with Tukey's multiple comparisons).

Increased anxiety and reduced working memory in CORTtreated mice; rescue by VFX in WT mice

CORT has been shown to increase anxiety as assessed by EPM testing in C57BL/6 mice, with rescue by fluoxetine. Herein we examine the effect of VFX on this endpoint. In addition, since increased $\gamma$ power has been linked to enhanced working memory (Alekseichuk et al., 2016; Goodman et al., 2018; Martorell et al., 2019), we also examine the effect of CORT and VFX on spontaneous alternations in the T-maze, which has been shown to measure spatial working memory in mice (Shoji et al., 2012).

Consistent with a reduction in anxiety-like behavior, VFX reduced closed-arm time in CORT-treated animals tested with the EPM (Fig. $7 A ; n=7-10$ mice per group; $F_{(2,21)}=3.88 p=$ 0.029; one-way ANOVA with Sidak's multiple comparisons testing). There was no significant difference between the CORT and CORT + VFX groups in MMP-9-null animals (Fig. $7 B ; n=7-10$ animals per group; $F_{(2,23)}=1.07 p=0.84$; ANOVA with Tukey's multiple comparisons testing). In Figure $7 C$, we examined the effects of CORT on T-maze performance. CORT decreased working memory in WT mice $\left(n=10\right.$ mice per group, $t_{(18)}=3.2$, $p=0.0049$, Student's $t$ test). We also examined working memory in WT and MMP-9-null mice treated with CORT or CORT + VFX (Fig. 7C,D). VFX improved T-maze performance in CORTtreated control ( $n=5$ mice per group; $F_{(3,16)}=4.6 p=0.045$; ANOVA with Sidak's multiple comparisons testing). In addition, VFX improved performance in animals that did not receive CORT ( $n=5$ mice per group, $p=0.045$, ANOVA with Sidak's multiple comparisons testing). The difference between control and CORT groups was not significant in this cohort, which may be due to injection stress in both groups and/or a smaller $n$. In addition, although the WT controls in Figure $7 C, D$ showed differential performance, it should be noted that controls in Figure $7 D$ received stressful daily intraperitoneal saline, whereas controls in Figure $7 C$ received cyclodextrin $(C D)$ in their drinking water. In Figure $7 E$, we show T-maze results for MMP-9-null 
A

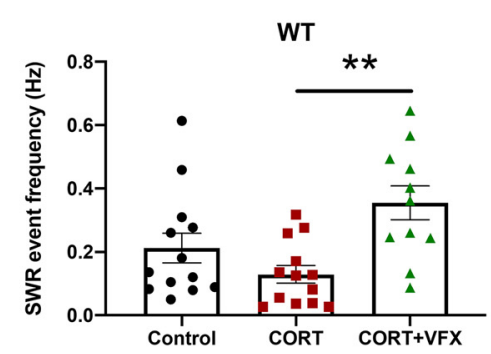

C

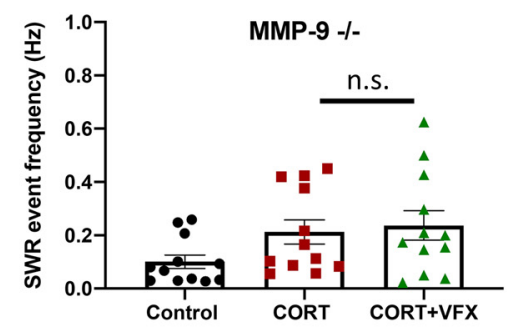

B
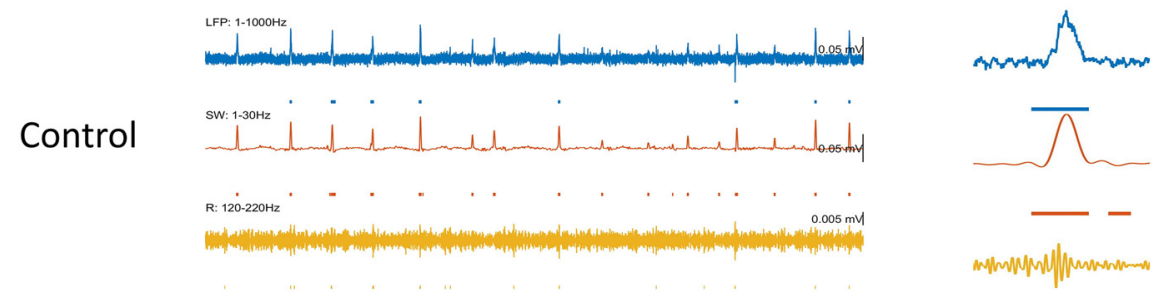

-

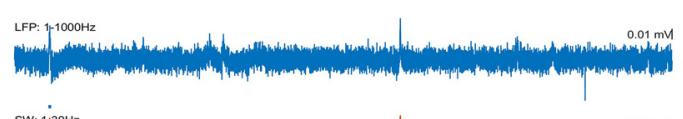

CORT
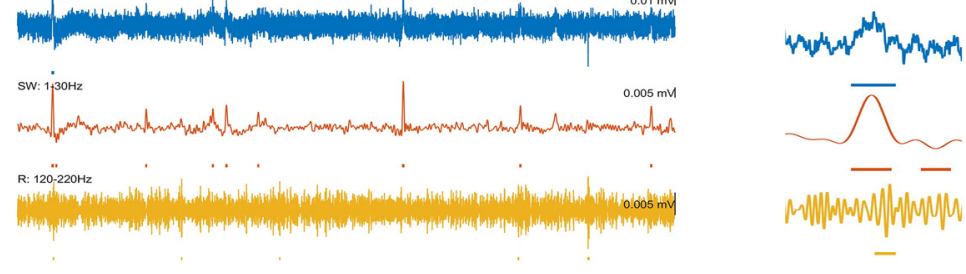

R: $120-220 \mathrm{~Hz}$
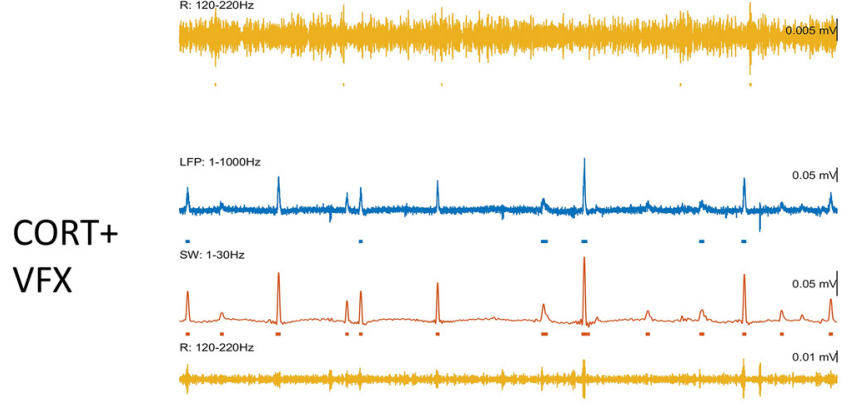

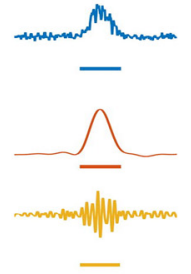

Figure 6. VFX stimulates an MMP-9-dependent increase in SWR abundance in ex vivo hippocampal slices from CORT-treated animals. $\boldsymbol{A}$, VFX increases SWR abundance in CORT-treated WT animals (4-6 mice, $n=11-13$ slices, $p=0.0025$, one-way ANOVA with Tukey's multiple comparisons). $\boldsymbol{B}$, Representative traces for WT animals. $\boldsymbol{C}$, In contrast, VFX did not significantly increase SWR abundance in CORT-treated MMP-9-null mice (4 or 5 mice, $n=12$ slices, $p=0.9$, one-way ANOVA with Tukey's multiple comparisons). ${ }^{* *} p<0.005$, n.S., non significant.

animals. We did not have a VFX only group for these animals. However, in contrast to WT animals, VFX did not improve performance in CORT-treated MMP-9-null mice $(n=7-10$ animals per group; $p=0.36 F_{(2,33)}=1.34 p=0.36$; ANOVA with Tukey's multiple comparisons testing).

\section{Mmp-9/TIMP-1 levels are increased in PFC samples from MDD patients treated with monoamine antidepressants} PNN alterations have been examined in depression and animal models of the same. Sulfation patterns that render nets more resistant to proteolysis have been observed in human postmortem tissue from depressed patients and increased PNN deposition has been noted in animal models of both stress and depression (Pantazopoulos et al., 2015; Riga et al., 2017). Brain levels of PNN modulating proteases have, however, not been well examined. To assess the possibility that MMP-9 levels may be altered with depression and/or increased by monoamine antidepressants, we analyzed postmortem PFC lysates from control patients, untreated MDD patients, and monoamine antidepressant-treated MDD patients for MMP-9 protein based on an ELISA. We also analyzed these lysates for levels of tissue inhibitor of metalloproteinase-1, an endogenous inhibitor of MMP-9. Results are shown in Figure $8 A, B$, and demonstrate that, compared with control patients, monoamine antidepressant-treated patients (antidepressant drug $[\mathrm{ADD}]$ ) have increased levels of MMP-9 in the PFC (Fig. $8 A ; F_{(2,3)}=4.19 p=0.0130$; one-way ANOVA with Dunnett's multiple comparisons; 2 high outliers in the MDD/ADD group were excluded). In contrast, there is no significant difference between MMP-9 levels in control and untreated MDD patients $(p=0.45)$. Moreover, there is a significant increase in the MMP-9/TIMP-1 ratio in monoamine antidepressant-treated MDD patients compared with the same ratio in controls or untreated MDD patients (Control vs MDD $+\mathrm{ADD} ; F_{(2,39)}=6.7 p=0.0052$; one-way ANOVA with Tukey's multiple comparisons; $\mathrm{MDD}$ vs $\mathrm{MDD}+\mathrm{ADD} ; p=0.009$, oneway ANOVA with Tukey's multiple comparisons).

Of note, there was no significant difference in MMP-9/TIMP1 ratio as a function of sex $(n=24$ males and 17 females, average MMP-9/TIMP-1 in males 7.82 \pm 0.87 SEM, average MMP-9/ TIMP- 1 in females $8.34 \pm 1.68$ SEM). Moreover, in postunblinding exploratory analyses, limited by a low $n$ due to cause of death ambiguity for some patients, we compared MMP-9/TIMP-1 ratios in MDD patients who committed suicide versus those who did not. The average MMP-9/TIMP-1 in suicide patients $(n=9)$ was $1.64 \pm 1.3$ SEM and the ratio in nonsuicide patients $(n=4)$ was $7.02 \pm 0.99$ SEM.

\section{Schematic; hypothetical changes in PNN deposition and MMP-9 activity in untreated and treated MDD}

Stress and depression can enhance PNN deposition and also render PNNs resistant to proteolysis (Pantazopoulos et al., 2015; Riga et al., 2017; Simard et al., 2018). Both processes can increase overall PNN levels. Monoamine reuptake inhibitors can, however, act to increase expression of PNN-degrading proteases and normalize PNN levels (Fig. 9). Although excess PNN remodeling may enhance neuronal plasticity at the cost of memory stability (Gogolla et al., 2009; Slaker et al., 2015; Carulli et al., 2016), moderately increased PNN remodeling in the background of excess deposition may be adaptive or therapeutic. Stress might also reduce dendritic arbor, through PNN-dependent and/or -independent mechanisms, and this may 
A

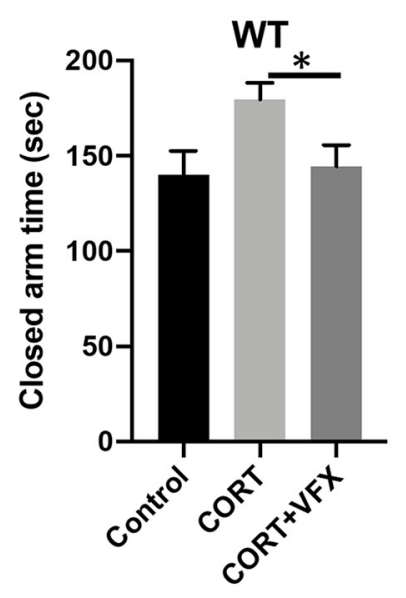

B

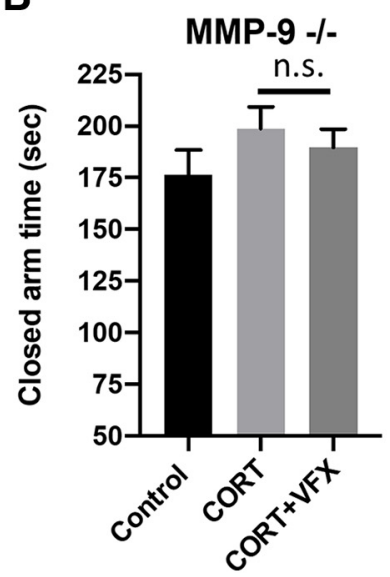

C

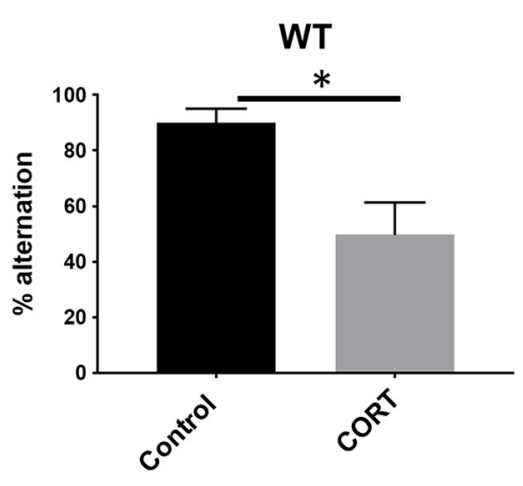

$\mathbf{E}$

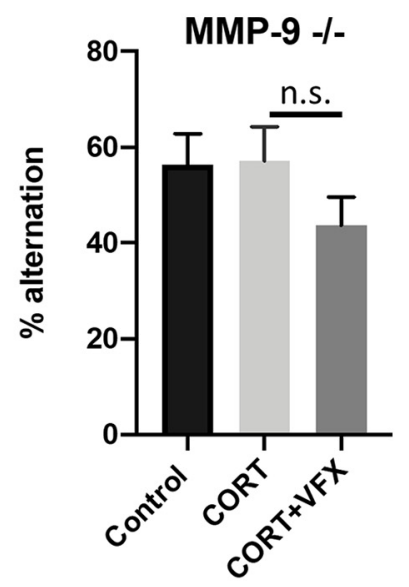

Figure 7. Reduced working memory in CORT-treated mice; rescue by VFX in WT mice. VFX reduced closed-arm time in CORT-treated animals tested with the EPM $(\boldsymbol{A}$; $n=7-10$ mice per group, $p=0.029$, one-way ANOVA with Sidak's multiple comparisons testing). There was no significant difference between the CORT and CORT + VFX groups in MMP-9-null animals ( $\boldsymbol{B}$; $n=7-10$ animals per group, $p=0.84$, ANOVA with Tukey's multiple comparisons testing). $\boldsymbol{C}$, We examined the effects of CORT on T-maze performance. CORT decreased working memory in WT mice ( $n=10$ mice per group, $p=0.0049$, Student's $t$ test). C, D, We also examined working memory in WT and MMP-9-null mice treated with CORT or CORT + VFX. VFX improved Tmaze performance in CORT-treated control ( $n=5$ mice per group, $p=0.045$, ANOVA with Sidak's multiple comparisons testing). In addition, VFX improved performance in animals that did not receive CORT ( $n=5$ mice per group, $p=0.045$, ANOVA with Sidak's multiple comparisons testing). The difference between control and CORT groups was not significant in this cohort, which may be due to injection stress in both groups and/or a smaller $n$. In addition, although the WT controls in $\boldsymbol{C}$ and $\boldsymbol{D}$ showed differential performance, it should be noted that controls in $\boldsymbol{D}$ received stressful daily intraperitoneal saline, whereas controls in $\boldsymbol{C}$ received CD in their drinking water. $\boldsymbol{E}$, T-maze results for MMP-9-null animals. We did not have a VFX only group for these animals. However, in contrast to WT animals, VFX did not improve performance in CORT-treated MMP-9-null mice ( $n=7-10$ animals per group, $p=0.36$, ANOVA with Tukey's multiple comparisons testing). ${ }^{*} p<0.05$, n.S., non significant.

be normalized by monoamine antidepressant associated changes in MMP-9 (data not shown).

\section{Discussion}

Herein, we tested the hypothesis that VFX stimulates MMP-9dependent changes relevant to increased E/I balance in conventionally housed and/or CORT-treated mice. We also tested an association between antidepressant use and MMP-9 levels in humans. Our hypothesis was based on prior work linking MMP9 to endpoints, including enhanced hippocampal-dependent learning and memory (Nagy et al., 2006), as well as monoaminestimulated MMP-9 activity (Bijata et al., 2017).

In work related to monoamines and MMP-9 release, a 5HT7 agonist stimulates MMP-9-dependent growth of dendritic spines in hippocampal cultures and slices (Bijata et al., 2017). 5HT7 is coupled to $\mathrm{G} \gamma_{\mathrm{s}}$, which increases cAMP levels and cfos-dependent transcription (Boutillier et al., 1992). The latter is a critical transcription factor for MMP-9 expression (Kaczmarek, 2018).
Serotonin reuptake inhibitors are also thought to act on $\mathrm{G} \gamma_{\mathrm{s}^{-}}$ coupled receptors to increase $\gamma$-secretase activity (Fisher et al., 2016). With ADAM-10, MMP-9 represents a potent $\gamma$-secretase (Fragkouli et al., 2012). Because VFX can increase both serotonin and norepinephrine signaling, we examined MMP-9 release from cultured hippocampal neurons stimulated with $\beta$-adrenergic agonists that activate $\mathrm{G} \gamma_{\mathrm{s}}$-coupled receptors. Isoproterenol indeed increased neuronal MMP-9 release.

In work more directly linked to our central hypotheses, VFX stimulated MMP-9-dependent increases in cortical dendritic arbor and hippocampal PSD-95 expression. Consistent with this, previous studies have shown that MMP-9 can induce spine head protrusions (Szepesi et al., 2013) in organotypic cultures, and spine expansion in hippocampal slices (Wang et al., 2008). Moreover, a pan-MMP inhibitor reduces neurite outgrowth in cultured cortical neurons (Ould-yahoui et al., 2009).

We acknowledge that the MMP-9 dependence of our findings is inferred from comparison with constitutive KOs. Although 
A

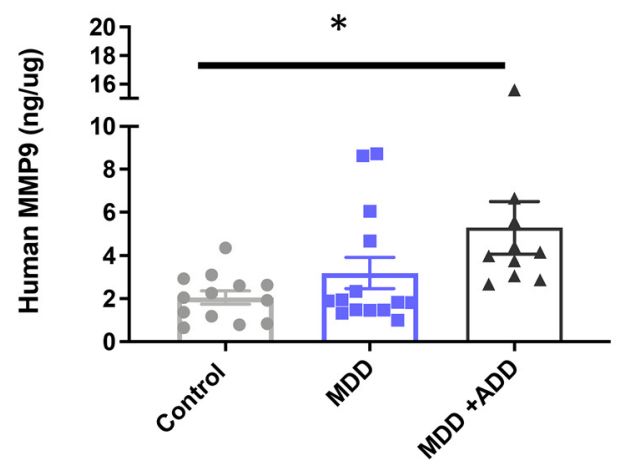

B

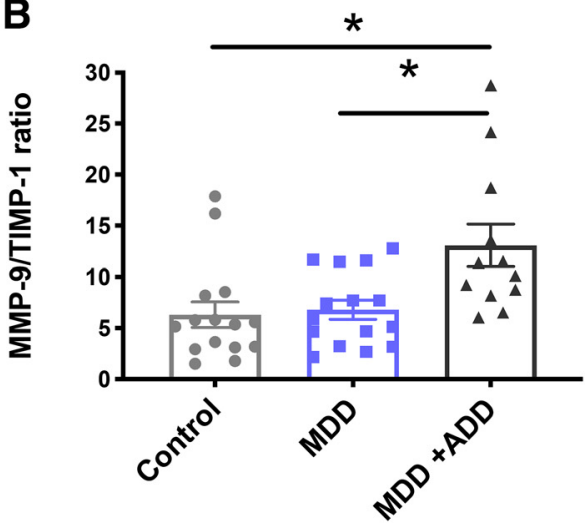

Figure 8. MMP-9/TIMP-1 levels are increased in PFC samples from MDD patients treated with monoamine antidepressants. $\boldsymbol{A}, \boldsymbol{B}$, Compared with control patients, monoamine antidepressant-treated patients (ADD) have increased levels of MMP-9 in the PFC ( $\boldsymbol{A}, \boldsymbol{p}=0.0130$, one-way ANOVA with Dunnett's multiple comparisons). In contrast, there is no significant difference between MMP-9 levels in control and untreated MDD patients $(p=0.45)$. Moreover, there is a significant increase in the MMP-9/TIMP-1 ratio in monoamine antidepressant-treated MDD patients compared with the same ratio in controls or untreated MDD patients (Control vs MDD + ADD, $p=0.0052$, one-way ANOVA with Tukey's multiple comparisons; MDD vs MDD + ADD, $p=0.009$, one-way ANOVA with Tukey's multiple comparisons). ${ }^{*} p<0.05$.

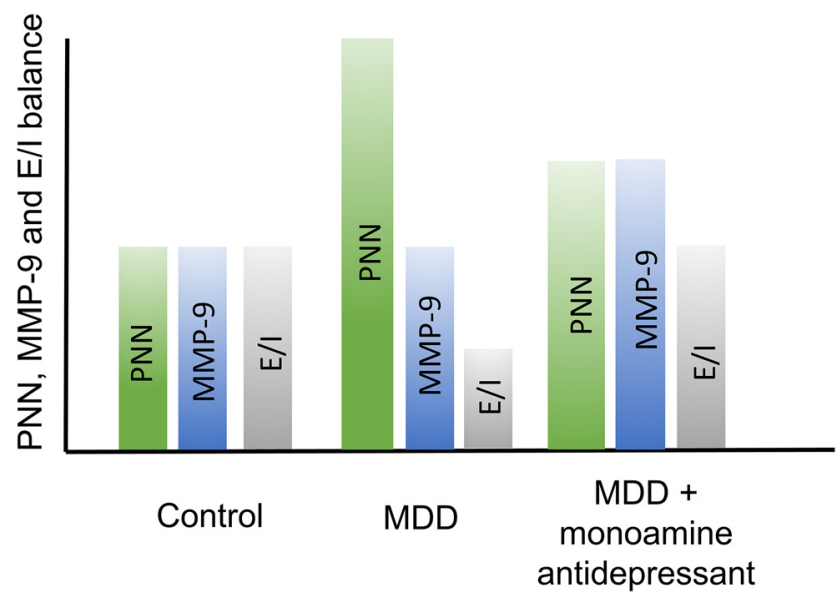

Figure 9. Hypothetical changes in PNN deposition and MMP-9 activity in untreated and treated MDD. Hypothetical PNN, MMP levels, and E/I balance (arbitrary units) are shown for nondepressed control patients. With MDD, PNN levels increase and thus E/l balance decreases. With MDD in the setting of antidepressant therapy, however, MMP levels also increase to potentially restore $\mathrm{E} / \mathrm{I}$ balance.

alternatives include the use of MMP inhibitors, these inhibitors are nonspecific (Fields, 2015). In the absence of inducible MMP9 KOs, constitutive KOs have demonstrated MMP-9-dependent LTP (Nagy et al., 2006). In contrast, MMP-9 KOs did not show differences in basic properties of synaptic transmission, such as stimulus/response properties of fEPSCs (Nagy et al., 2006). Constitutive KOs have also demonstrated MMP-9-dependent deficits in a mouse model of Fragile X syndrome and MMP-9dependent plasticity following light reintroduction after dark (Murase et al., 2017; Wen et al., 2018). Consistent with prior studies (Tian et al., 2007), baseline differences in PSD-95 between MMP-9 KOs and controls were not observed in adult animals. Baseline differences in somatosensory dendritic arbor could not be compared, since WT animals with and without VFX were stained at one time and KOs at another. Dendritic arbor results were, however, normalized to the appropriate control.

We also observed an MMP-9-dependent reduction in overall PNN intensity and PV/PNN ratios in WT animals. MMP-9 KOs also demonstrated reduced basal and VFX-stimulated cleavage of BCAN while levels of full-length BCAN were not reduced in MMP-9 KOs. One possibility is that BCAN turnover is increased in WT compared with MMP-9-null mice. Specifics of PNN composition and/or turnover may be functionally relevant (Carulli et al., 2010; Sorg et al., 2016). Cleaved BCAN may function differently than full-length, and newly synthesized BCAN could be poorly localized.

The MMP-9 dependence of basal and VFX-associated changes in BCAN cleavage is interesting given that PNN enwrapped PV neurons are not noted to express MMP-9 under basal conditions (Rossier et al., 2015). MMP-9 could be released from excitatory inputs to PV-expressing neurons. Indeed, MMPs have been noted at synaptic boutons (Shilts and Broadie, 2017).

We additionally observed that BCAN levels and PNN staining were increased in the CORT model of depression, with the latter normalized by VFX in WT mice. These results are consistent with those from a study in a social defeat stress in rats, in which levels of PNN components were increased (Riga et al., 2017). Imipramine normalized stress-associated changes in PNN, although underlying molecular mechanisms were not examined (Riga et al., 2017). Since PNN reductions are linked to pyramidal cell disinhibition (Hayani et al., 2018), our work supports a link between monoamine reuptake inhibition and increased in $\mathrm{E} / \mathrm{I}$ balance.

We thus tested $\mathrm{E} / \mathrm{I}$ balance in ex vivo murine slices using an approach based on neuronal population activity. Population recordings have the advantage of detecting relatively broad changes in E/I balance, and may also detect alterations if PNN disruption is limited to a percentage of cells. Changes in singlecell recordings may instead depend on whether recordings are made in specific cells in which PNN changes are appreciable. Consistent with the possibility that increased PNN deposition enhances PV interneuron-mediated inhibition, CORT treatment reduced $\gamma$ power in ex vivo slices in WT mice. Moreover, VFX stimulated an MMP-9-dependent increase in SWR abundance and carbachol-stimulated $\gamma$ power in ex vivo hippocampal slices from CORT-treated WT but not MMP-9-null animals.

$\gamma$ power represents a physiological correlate of increased E/I balance and is critical to memory (Yizhar et al., 2011; Zheng et al., 2016; Lensjo et al., 2017). Increased hippocampal $\gamma$ power improves pattern completion and the quality of retrieved spatial memories (Staresina et al., 2016; Stevenson et al., 2018). 
Increases in theta and $\gamma$ power during encoding also predict recall (Sederberg et al., 2003). $\gamma$ oscillations are critical to working memory (Yamamoto et al., 2014), which may be limited by the number of $\gamma$ cycles nested within a theta cycle (Lisman, 2010). VFX's enhancement of $\gamma$ power is relevant to depression since $\gamma$ power is altered in this condition (Liu et al., 2012) and increased with remission in both humans and animal models (Khalid et al., 2016; Arikan et al., 2018). Moreover, increased $\gamma$ power correlates with improvements on the Hamilton Depression Scale (Arikan et al., 2018).

VFX's enhancement of SWR abundance is also relevant to depression. During SWRs, cell assemblies that were sequentially activated during encoding are sequentially reactivated in a timecompressed manner during offline states. SWRs are critical to memory consolidation (Ego-Stengel and Wilson, 2010; Buzsaki, 2015; Jones et al., 2019), which may be impaired in MDD (MacQueen and Frodl, 2011). Further study on the quality of SWRs in terms of reactivation of learning-relevant assemblies, as opposed to random assemblies, are warranted.

Since PV fast spiking interneuron activity is critical to $\gamma$ and ripple expression, it is of interest that, despite reduced $\mathrm{PV}$ activity with PNN attenuation (Hayani et al., 2018; Tewari et al., 2018), $\gamma$ oscillations and SWR events are not only maintained but increased in power and event frequency. This is consistent with the possibility that the PV population retains responsivity when excitatory input is strong, and is supported by previous work (Balmer, 2016).

A caveat is that MMP-9 has substrates in addition to PNN components that may increase pyramidal cell activity and thus $\gamma$ power or SWR abundance through relatively direct effects on excitatory neurons. These mechanisms include rapid MMP-dependent generation of integrin-binding ligands and integrinstimulated expansion of spines on glutamatergic neurons (Lonskaya et al., 2013). In addition, a small population of pyramidal neurons in CA2 hippocampus are PNN-enveloped, which may restrict their ability to undergo LTP (Carstens et al., 2016). However, given the abundance of the PV neuron-associated $\mathrm{PNN}$ and the potential for each $\mathrm{PV}^{+}$cell to impact a number of pyramidal cells, we posit that disruption of PV neuron-associated PNNs makes a strong contribution to these endpoints in our study. Future studies with mutants that render nets relatively resistant to proteolysis may be useful for additional support (Foscarin et al., 2017).

Previous work has examined effects of monoamine antidepressants on long-term memory (Van Dyke et al., 2019). Because of the link between $\gamma$ power and working memory, behavioral studies herein focused on short-term memory. T-maze performance was reduced with CORT and enhanced by VFX in conventionally housed and CORT-treated mice. In contrast, VFX did not enhance working memory in MMP-9-null mice. These results are consistent with the effects of the PNN-degrading enzymes on object place recognition (Riga et al., 2017) and reversal learning (Happel et al., 2014).

Consistent with the possibility that monoamine antidepressants stimulate MMP-9 expression in humans, we observed increased levels of MMP-9 and increased MMP-9/TIMP-1 ratios in antidepressant-treated individuals. TIMP-1 is inducible and expressed in areas relevant to depression. TIMP-1 inhibits MMP-9 to a greater degree than does TIMP-2; moreover, TIMP1 abolishes MMP-9-dependent LTP in PFC (Okulski et al., 2007). Our initial hypothesis was that MMP-9 levels would be lower in the depressed group and normalized by antidepressant treatment. The observation that treated patients have higher
MMP-9/TIMP-1 ratios than control or depressed individuals suggests that changes occurring with depression, such as increased PNN deposition, may instead be normalized by treatment-associated increases in MMP-9.

In conclusion, our study demonstrates that VFX stimulates MMP-9-dependent increases in dendritic arbor and PSD-95 expression. We are also the first to show MMP-9-dependent changes in ex vivo $\gamma$ power, SWR abundance, and working memory in a CORT model of depression. Future studies may address the question of whether these changes are largely beneficial and whether agents that target PNN integrity and/or $\gamma$ power, such as GENUS (Martorell et al., 2019), might also be useful for the treatment of depression.

\section{References}

Alaiyed S, Bozzelli PL, Caccavano A, Wu JY, Conant K (2019) Venlafaxine stimulates PNN proteolysis and MMP-9-dependent enhancement of gamma power: relevance to antidepressant efficacy. J Neurochem 148:810-821.

Alekseichuk I, Turi Z, Amador de Lara G, Antal A, Paulus W (2016) Spatial working memory in humans depends on theta and high gamma synchronization in the prefrontal cortex. Curr Biol 26:1513-1521.

Allen M, Ghosh S, Ahern GP, Villapol S, Maguire-Zeiss KA, Conant K (2016) Protease induced plasticity: matrix metalloproteinase-1 promotes neurostructural changes through activation of protease activated receptor 1. Sci Rep 6:35497.

Arikan MK, Metin B, Tarhan N (2018) EEG gamma synchronization is associated with response to paroxetine treatment. J Affect Disord 235:114116.

Balmer TS (2016) Perineuronal nets enhance the excitability of fast-spiking neurons. eNeuro 3:ENEURO.0112-16.2016.

Barber J (2011) Examining the use of tramadol hydrochloride as an antidepressant. Exp Clin Psychopharmacol 19:123-130.

Beaudoin GM, Lee SH, Singh D, Yuan Y, Ng YG, Reichardt LF, Arikkath J (2012) Culturing pyramidal neurons from the early postnatal mouse hippocampus and cortex. Nat Protoc 7:1741-1754.

Belmaker RH, Agam G (2008) Major depressive disorder. N Engl J Med 358:55-68.

Berger S, Gureczny S, Reisinger SN, Horvath O, Pollak DD (2019) Effect of chronic corticosterone treatment on depression-like behavior and sociability in female and male C57BL/6N mice. Cells 8:E1018.

Bijata M, Labus J, Guseva D, Stawarski M, Butzlaff M, Dzwonek J, Schneeberg J, Böhm K, Michaluk P, Rusakov DA, Dityatev A, Wilczyński G, Wlodarczyk J, Ponimaskin E (2017) Synaptic remodeling depends on signaling between serotonin receptors and the extracellular matrix. Cell Rep 19:1767-1782.

Boutillier AL, Barthel F, Roberts JL, Loeffler JP (1992) Beta-adrenergic stimulation of $\mathrm{cFOS}$ via protein kinase $\mathrm{A}$ is mediated by cAMP regulatory element binding protein (CREB)-dependent and tissue-specific CREBindependent mechanisms in corticotrope cells. J Biol Chem 267:2352023526.

Buzsaki G (2015) Hippocampal sharp wave-ripple: a cognitive biomarker for episodic memory and planning. Hippocampus 25:1073-1188.

Carstens KE, Phillips ML, Pozzo-Miller L, Weinberg RJ, Dudek SM (2016) Perineuronal nets suppress plasticity of excitatory synapses on CA2 pyramidal neurons. J Neurosci 36:6312-6320.

Carulli D, Kwok JC, Pizzorusso T (2016) Perineuronal nets and CNS plasticity and repair. Neural Plast 2016:1-2.

Carulli D, Pizzorusso T, Kwok JC, Putignano E, Poli A, Forostyak S, Andrews MR, Deepa SS, Glant TT, Fawcett JW (2010) Animals lacking link protein have attenuated perineuronal nets and persistent plasticity. Brain 133:2331-2347.

Conant K, Wang Y, Szklarczyk A, Dudak A, Mattson MP, Lim ST (2010) Matrix metalloproteinase-dependent shedding of intercellular adhesion molecule-5 occurs with long-term potentiation. Neuroscience 166:508521.

David DJ, Samuels BA, Rainer Q, Wang JW, Marsteller D, Mendez I, Drew M, Craig DA, Guiard BP, Guilloux JP, Artymyshyn RP, Gardier AM, Gerald C, Antonijevic IA, Leonardo ED, Hen R (2009) Neurogenesis- 
dependent and -independent effects of fluoxetine in an animal model of anxiety/depression. Neuron 62:479-493.

Ego-Stengel V, Wilson MA (2010) Disruption of ripple-associated hippocampal activity during rest impairs spatial learning in the rat. Hippocampus 20:1-10.

Fales CL, Barch DM, Rundle MM, Mintun MA, Mathews J, Snyder AZ, Sheline YI (2009) Antidepressant treatment normalizes hypoactivity in dorsolateral prefrontal cortex during emotional interference processing in major depression. J Affect Disord 112:206-211.

Favuzzi E, Marques-Smith A, Deogracias R, Winterflood CM, SanchezAguilera A, Mantoan L, Maeso P, Fernandes C, Ewers H, Rico B (2017) Activity-dependent gating of parvalbumin interneuron function by the perineuronal net protein brevican. Neuron 95:639-655.e610.

Fawcett JW, Oohashi T, Pizzorusso T (2019) The roles of perineuronal nets and the perinodal extracellular matrix in neuronal function. Nat Rev Neurosci 20:451-465.

Fields GB (2015) New strategies for targeting matrix metalloproteinases. Matrix Biol 44-46:239-246.

Fisher JR, Wallace CE, Tripoli DL, Sheline YI, Cirrito JR (2016) Redundant Gs-coupled serotonin receptors regulate amyloid-beta metabolism in vivo. Mol Neurodegener 11:45.

Foscarin S, Raha-Chowdhury R, Fawcett JW, Kwok JC (2017) Brain ageing changes proteoglycan sulfation, rendering perineuronal nets more inhibitory. Aging 9:1607-1622.

Fragkouli A, Papatheodoropoulos C, Georgopoulos S, Stamatakis A, Stylianopoulou F, Tsilibary EC, Tzinia AK (2012) Enhanced neuronal plasticity and elevated endogenous sAPPalpha levels in mice overexpressing MMP9. J Neurochem 121:239-251.

Frischknecht R, Heine M, Perrais D, Seidenbecher CI, Choquet D, Gundelfinger ED (2009) Brain extracellular matrix affects AMPA receptor lateral mobility and short-term synaptic plasticity. Nat Neurosci 12:897-904.

Ganguly K, Rejmak E, Mikosz M, Nikolaev E, Knapska E, Kaczmarek L (2013) Matrix metalloproteinase (MMP) 9 transcription in mouse brain induced by fear learning. J Biol Chem 288:20978-20991.

Gogolla N, Caroni P, Luthi A, Herry C (2009) Perineuronal nets protect fear memories from erasure. Science 325:1258-1261.

Goodman MS, Kumar S, Zomorrodi R, Ghazala Z, Cheam AS, Barr MS, Daskalakis ZJ, Blumberger DM, Fischer C, Flint A, Mah L, Herrmann N, Bowie CR, Mulsant BH, Rajji TK (2018) Theta-gamma coupling and working memory in Alzheimer's dementia and mild cognitive impairment. Front Aging Neurosci 10:101.

Happel MF, Niekisch H, Castiblanco Rivera LL, Ohl FW, Deliano M, Frischknecht R (2014) Enhanced cognitive flexibility in reversal learning induced by removal of the extracellular matrix in auditory cortex. Proc Natl Acad Sci USA 111:2800-2805.

Hayani H, Song I, Dityatev A (2018) Increased excitability and reduced excitatory synaptic input into fast-spiking CA2 interneurons after enzymatic attenuation of extracellular matrix. Front Cell Neurosci 12:149.

Jones EA, Gillespie AK, Yoon SY, Frank LM, Huang Y (2019) Early hippocampal sharp-wave ripple deficits predict later learning and memory impairments in an Alzheimer's disease mouse model. Cell Rep 29:21232133.e24.

Kaczmarek L (2018) From c-Fos to MMP-9: in control of synaptic plasticity to produce healthy and diseased mind, a personal view. Postepy Biochem 64:101-109.

Kallarackal AJ, Kvarta MD, Cammarata E, Jaberi L, Cai X, Bailey AM, Thompson SM (2013) Chronic stress induces a selective decrease in AMPA receptor-mediated synaptic excitation at hippocampal temporoammonic-CA1 synapses. J Neurosci 33:15669-15674.

Kang L, Zhang A, Sun N, Liu P, Yang C, Li G, Liu Z, Wang Y, Zhang K (2018) Functional connectivity between the thalamus and the primary somatosensory cortex in major depressive disorder: a resting-state fMRI study. BMC Psychiatry 18:339.

Khalid A, Kim BS, Seo BA, Lee ST, Jung KH, Chu K, Lee SK, Jeon D (2016) Gamma oscillation in functional brain networks is involved in the spontaneous remission of depressive behavior induced by chronic restraint stress in mice. BMC Neurosci 17:4.

Kropf E, Syan SK, Minuzzi L, Frey BN (2019) From anatomy to function: the role of the somatosensory cortex in emotional regulation. Braz J Psychiatry 41:261-269.
Lee R, Kermani P, Teng KK, Hempstead BL (2001) Regulation of cell survival by secreted proneurotrophins. Science 294:1945-1948.

Lensjo KK, Lepperod ME, Dick G, Hafting T, Fyhn M (2017) Removal of perineuronal nets unlocks juvenile plasticity through network mechanisms of decreased inhibition and increased gamma activity. J Neurosci 37:1269-1283.

Li Y, Partridge J, Berger C, Sepulveda-Rodriguez A, Vicini S, Conant K (2016) Dopamine increases NMDA-stimulated calcium flux in striatopallidal neurons through a matrix metalloproteinase-dependent mechanism. Eur J Neurosci 43:194-203.

Lisman J (2010) Working memory: the importance of theta and gamma oscillations. Curr Biol 20:R490-R492.

Liston C, Miller MM, Goldwater DS, Radley JJ, Rocher AB, Hof PR, Morrison JH, McEwen BS (2006) Stress-induced alterations in prefrontal cortical dendritic morphology predict selective impairments in perceptual attentional set-shifting. J Neurosci 26:7870-7874.

Liu TY, Hsieh JC, Chen YS, Tu PC, Su TP, Chen LF (2012) Different patterns of abnormal gamma oscillatory activity in unipolar and bipolar disorder patients during an implicit emotion task. Neuropsychologia 50:15141520.

Lonskaya I, Partridge J, Lalchandani RR, Chung A, Lee T, Vicini S, Hoe HS, Lim ST, Conant K (2013) Soluble ICAM-5, a product of activity dependent proteolysis, increases mEPSC frequency and dendritic expression of GluA1. PLoS One 8:e69136.

MacQueen G, Frodl T (2011) The hippocampus in major depression: evidence for the convergence of the bench and bedside in psychiatric research. Mol Psychiatry 16:252-264.

Magarinos AM, McEwen BS (1995) Stress-induced atrophy of apical dendrites of hippocampal CA3c neurons: involvement of glucocorticoid secretion and excitatory amino acid receptors. Neuroscience 69:89-98.

Martorell AJ, Paulson AL, Suk HJ, Abdurrob F, Drummond GT, Guan W, Young JZ, Kim DN, Kritskiy O, Barker SJ, Mangena V, Prince SM, Brown EN, Chung K, Boyden ES, Singer AC, Tsai LH (2019) Multi-sensory gamma stimulation ameliorates Alzheimer's-associated pathology and improves cognition. Cell 177:256-271.e22.

Meighan PC, Meighan SE, Davis CJ, Wright JW, Harding JW (2007) Effects of matrix metalloproteinase inhibition on short- and long-term plasticity of Schaffer collateral/CA1 synapses. J Neurochem 102:2085-2096.

Mekiri M, Gardier AM, David DJ, Guilloux JP (2017) Chronic corticosterone administration effects on behavioral emotionality in female c57bl6 mice. Exp Clin Psychopharmacol 25:94-104.

Millan MJ, Gobert A, Rivet JM, Adhumeau-Auclair A, Cussac D, NewmanTancredi A, Dekeyne A, Nicolas JP, Lejeune F (2000) Mirtazapine enhances frontocortical dopaminergic and corticolimbic adrenergic, but not serotonergic, transmission by blockade of alpha2-adrenergic and serotonin2C receptors: a comparison with citalopram. Eur J Neurosci 12:1079-1095

Murase S, Lantz CL, Quinlan EM (2017) Light reintroduction after dark exposure reactivates plasticity in adults via perisynaptic activation of MMP9. Elife 6:e27345.

Nagy V, Bozdagi O, Matynia A, Balcerzyk M, Okulski P, Dzwonek J, Costa RM, Silva AJ, Kaczmarek L, Huntley GW (2006) Matrix metalloproteinase- 9 is required for hippocampal late-phase long-term potentiation and memory. J Neurosci 26:1923-1934.

Nissen C, Holz J, Blechert J, Feige B, Riemann D, Voderholzer U, Normann C (2010) Learning as a model for neural plasticity in major depression. Biol Psychiatry 68:544-552.

Okulski P, Jay TM, Jaworski J, Duniec K, Dzwonek J, Konopacki FA, Wilczynski GM, Sanchez-Capelo A, Mallet J, Kaczmarek L (2007) TIMP1 abolishes MMP-9-dependent long-lasting long-term potentiation in the prefrontal cortex. Biol Psychiatry 62:359-362.

Ould-yahoui A, Tremblay E, Sbai O, Ferhat L, Bernard A, Charrat E, Gueye Y, Lim NH, Brew K, Risso JJ, Dive V, Khrestchatisky M, Rivera S (2009) A new role for TIMP-1 in modulating neurite outgrowth and morphology of cortical neurons. PLoS One 4:e8289.

Page CE, Coutellier L (2019) Prefrontal excitatory/inhibitory balance in stress and emotional disorders: evidence for over-inhibition. Neurosci Biobehav Rev 105:39-51.

Page CE, Shepard R, Heslin K, Coutellier L (2019) Prefrontal parvalbumin cells are sensitive to stress and mediate anxiety-related behaviors in female mice. Sci Rep 9:19772. 
Pantazopoulos H, Markota M, Jaquet F, Ghosh D, Wallin A, Santos A, Caterson B, Berretta S (2015) Aggrecan and chondroitin-6-sulfate abnormalities in schizophrenia and bipolar disorder: a postmortem study on the amygdala. Transl Psychiatry 5:e496.

Pawlak R, Rao BS, Melchor JP, Chattarji S, McEwen B, Strickland S (2005) Tissue plasminogen activator and plasminogen mediate stress-induced decline of neuronal and cognitive functions in the mouse hippocampus. Proc Natl Acad Sci USA 102:18201-18206.

Radley JJ, Rocher AB, Rodriguez A, Ehlenberger DB, Dammann M, McEwen BS, Morrison JH, Wearne SL, Hof PR (2008) Repeated stress alters dendritic spine morphology in the rat medial prefrontal cortex. J Comp Neurol 507:1141-1150.

Riga D, Kramvis I, Koskinen MK, van Bokhoven P, van der Harst JE, Heistek TS, Timmerman A, van Nierop P, van der Schors RC, Pieneman AW, de Weger A, van Mourik Y, Schoffelmeer AN, Mansvelder HD, Meredith RM, Hoogendijk WJ, Smit AB, Spijker S (2017) Hippocampal extracellular matrix alterations contribute to cognitive impairment associated with a chronic depressive-like state in rats. Sci Transl Med 9:eaai8753.

Rossier J, Bernard A, Cabungcal JH, Perrenoud Q, Savoye A, Gallopin T, Hawrylycz M, Cuenod M, Do K, Urban A, Lein ES (2015) Cortical fastspiking parvalbumin interneurons enwrapped in the perineuronal net express the metallopeptidases Adamts8, Adamts15 and Neprilysin. Mol Psychiatry 20:154-161.

Sederberg PB, Kahana MJ, Howard MW, Donner EJ, Madsen JR (2003) Theta and gamma oscillations during encoding predict subsequent recall. J Neurosci 23:10809-10814.

Shilts J, Broadie K (2017) Secreted tissue inhibitor of matrix metalloproteinase restricts trans-synaptic signaling to coordinate synaptogenesis. J Cell Sci 130:2344-2358.

Shoji H, Hagihara H, Takao K, Hattori S, Miyakawa T (2012) T-maze forced alternation and left-right discrimination tasks for assessing working and reference memory in mice. J Vis $\operatorname{Exp} 2012: 3300$.

Simard S, Coppola G, Rudyk CA, Hayley S, McQuaid RJ, Salmaso N (2018) Profiling changes in cortical astroglial cells following chronic stress. Neuropsychopharmacology 43:1961-1971.

Slaker M, Churchill L, Todd RP, Blacktop JM, Zuloaga DG, Raber J, Darling RA, Brown TE, Sorg BA (2015) Removal of perineuronal nets in the medial prefrontal cortex impairs the acquisition and reconsolidation of a cocaine-induced conditioned place preference memory. J Neurosci 35:4190-4202.

Smith AC, Kupchik YM, Scofield MD, Gipson CD, Wiggins A, Thomas CA, Kalivas PW (2014) Synaptic plasticity mediating cocaine relapse requires matrix metalloproteinases. Nat Neurosci 17:1655-1657.

Sorg BA, Berretta S, Blacktop JM, Fawcett JW, Kitagawa H, Kwok JC, Miquel M (2016) Casting a wide net: role of perineuronal nets in neural plasticity. J Neurosci 36:11459-11468.

St Hillaire C, Vargas D, Pardo CA, Gincel D, Mann J, Rothstein JD, McArthur JC, Conant K (2005) Aquaporin 4 is increased in association with human immunodeficiency virus dementia: implications for disease pathogenesis. J Neurovirol 11:535-543.
Staresina BP, Michelmann S, Bonnefond M, Jensen O, Axmacher N, Fell J (2016) Hippocampal pattern completion is linked to gamma power increases and alpha power decreases during recollection. Elife 5:e17397.

Stevenson RF, Zheng J, Mnatsakanyan L, Vadera S, Knight RT, Lin JJ, Yassa MA (2018) Hippocampal CA1 gamma power predicts the precision of spatial memory judgments. Proc Natl Acad Sci USA 115:10148-10153.

Szepesi Z, Bijata M, Ruszczycki B, Kaczmarek L, Wlodarczyk J (2013) Matrix metalloproteinases regulate the formation of dendritic spine head protrusions during chemically induced long-term potentiation. PLoS One 8: e63314.

Tewari BP, Chaunsali L, Campbell SL, Patel DC, Goode AE, Sontheimer H (2018) Perineuronal nets decrease membrane capacitance of peritumoral fast spiking interneurons in a model of epilepsy. Nat Commun 9:4724.

Thompson SM, Kallarackal AJ, Kvarta MD, Van Dyke AM, LeGates TA, Cai $\mathrm{X}$ (2015) An excitatory synapse hypothesis of depression. Trends Neurosci 38:279-294.

Tian L, Stefanidakis M, Ning L, Van Lint P, Nyman-Huttunen H, Libert C, Itohara S, Mishina M, Rauvala H, Gahmberg CG (2007) Activation of NMDA receptors promotes dendritic spine development through MMPmediated ICAM-5 cleavage. J Cell Biol 178:687-700.

Van Dyke AM, Francis TC, Chen H, Bailey AM, Thompson SM (2019) Chronic fluoxetine treatment in vivo enhances excitatory synaptic transmission in the hippocampus. Neuropharmacology 150:38-45.

Verslegers M, Van Hove I, Dekeyster E, Gantois I, Hu TT, D'Hooge R, Arckens L, Moons L (2015) MMP-2 mediates Purkinje cell morphogenesis and spine development in the mouse cerebellum. Brain Struct Funct 220:1601-1617.

Wang XB, Bozdagi O, Nikitczuk JS, Zhai ZW, Zhou Q, Huntley GW (2008) Extracellular proteolysis by matrix metalloproteinase-9 drives dendritic spine enlargement and long-term potentiation coordinately. Proc Natl Acad Sci USA 105:19520-19525.

Wen TH, Afroz S, Reinhard SM, Palacios AR, Tapia K, Binder DK, Razak KA, Ethell IM (2018) Genetic reduction of matrix metalloproteinase-9 promotes formation of perineuronal nets around parvalbumin-expressing interneurons and normalizes auditory cortex responses in developing Fmr1 knock-out mice. Cereb Cortex 28:3951-3964.

Yamamoto J, Suh J, Takeuchi D, Tonegawa S (2014) Successful execution of working memory linked to synchronized high-frequency gamma oscillations. Cell 157:845-857.

Yizhar O, Fenno LE, Prigge M, Schneider F, Davidson TJ, O'Shea DJ, Sohal VS, Goshen I, Finkelstein J, Paz JT, Stehfest K, Fudim R, Ramakrishnan C, Huguenard JR, Hegemann P, Deisseroth K (2011) Neocortical excitation/inhibition balance in information processing and social dysfunction. Nature 477:171-178.

Yohn CN, Ashamalla SA, Bokka L, Gergues MM, Garino A, Samuels BA (2019) Social instability is an effective chronic stress paradigm for both male and female mice. Neuropharmacology 160:107780.

Zheng C, Bieri KW, Hwaun E, Colgin LL (2016) Fast gamma rhythms in the hippocampus promote encoding of novel object-place pairings. eNeuro 3:ENEURO.0001-16.2016. 